\title{
Extended radio emission in BL Lac objects
}

\author{
I. The images

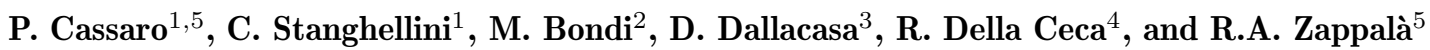 \\ 1 Istituto di Radioastronomia del CNR, C.P. 141, I-96017 Noto SR, Italy \\ 2 Istituto di Radioastronomia del CNR, Via Gobetti 101, I-40129 Bologna, Italy \\ 3 Dipartimento di Astronomia, Università di Bologna, Via Ranzani 1, I-40127, Bologna, Italy \\ 4 Osservatorio Astronomico di Brera, Via Brera 28, I-20121 Milano, Italy \\ 5 Istituto di Astronomia dell'Università, Città Universitaria, viale Andrea Doria 6, I-95125 Catania, Italy
}

Received March 24; accepted July 28, 1999

\begin{abstract}
We have observed 28 sources selected from the 1Jy sample of BL Lac objects (Stickel et al. 1991) with the Very Large Array (VLA) in A, B and D configurations at $1.36,1.66$ and $4.85 \mathrm{GHz}$, and/or with the Westerbork Synthesis Radio Telescope (WSRT) at $1.40 \mathrm{GHz}$. In this paper we present high sensitivity images at arcsecond resolution of the 18 objects showing extended structure in our images, and of another source from the FIRST (Faint Images of the Radio Sky at Twenty-cm) survey (Becker et al. 1995). In general our high sensitivity images reveal an amount of extended emission larger than previously reported. In some objects the luminosity of the extended structure is comparable with that of FR II radio sources. A future paper will be devoted to the interpretation of these results.
\end{abstract}

Key words: galaxies: active — BL Lac objects: general — radio continuum: galaxies

\section{Introduction}

A great effort of the recent research on Active Galactic Nuclei (AGN) has been directed to the development of "Unified Schemes": a framework wherein the observational properties of different classes of AGN can be explained as intrinsically similar objects seen at different orientation angles to the line of sight (see Urry \& Padovani 1995 and references therein).

In this context, it is now widely accepted that the observed properties of BL Lac objects are largely due to a relativistic jet pointing in the direction of the observer

Send offprint requests to: P. Cassaro,

e-mail: cassaro@ira.noto.cnr.it (the Beaming Model; Blandford \& Rees 1978), implying the existence of a class of radio sources (hereafter the parent population) intrinsically identical to BL Lacs, but with the jets oriented at large angles with respect to the line of sight.

The nature of the parent population of the BL Lacs has been the subject of several investigations in the past. While some authors confirm that the majority of them are low luminosity edge darkened FR I radio galaxies seen along their radio axis (see Urry \& Padovani 1995 and references therein), others suggest that the diffuse radio emission detected around high redshift BL Lacs is more consistent with FR II rather than FR I radio galaxies (see e.g. Kollgaard et al. 1992; Murphy et al. 1993).

A straightforward outcome of the beaming model is that all the properties not depending on orientation should be shared by the BL Lac objects and their parent population.

To address this problem we planned to compare the extended radio luminosity of the radio selected 1 Jy BL Lac sample (Stickel et al. 1991) with that of FR I and FR II radio galaxies. In fact, while the morphology is distorted by projection effects, and a morphological classification could be uncertain, the luminosity of the unbeamed emission is suitable for a direct comparison with the extended emission found in the candidate parent population.

Of the 34 BL Lac objects belonging to the 1 Jy sample we have selected the 28 objects without deep radio images at arcsecond scale resolution in the literature, and/or at the arcminute scale. Stickel et al. (1994) added three sources to their original sample: $0218+357,2029+121$ and $2150+173$. In particular $0218+357$ is a well known gravitational lens (O'Dea et al. 1992; Patnaik et al. 1993, 1995). Since our work started when these three additional sources were not included, and furthermore, in the literature the "1 Jy sample of BL Lac sources" refers to the 34 objects 
Table 1. Journal of the observations: Col. [1] date of the observations, Col. [2] array (A, B, D, for the VLA configurations, W for WSRT), Col. [3] observing frequency, Col. [4] bandwidth

\begin{tabular}{cccc}
\hline \hline date & array & $\nu(\mathrm{GHz})$ & $\Delta \nu(\mathrm{MHz})$ \\
\hline $18 / 04 / 94$ & A & 1.36 & 50 \\
& A & 1.66 & 25 \\
$29 / 04 / 94$ & A & 1.36 & 50 \\
& A & 1.66 & 25 \\
$04 / 07 / 94$ & B & 1.36 & 50 \\
& B & 1.66 & 25 \\
$22 / 01 / 95$ & D & 4.88 & $50 \times 2$ IFs \\
& D & 1.36 & 50 \\
$21 / 02 / 95$ & D & 1.36 & 25 \\
& D & 1.66 & 25 \\
$02 / 94$ & W & 1.40 & 40 \\
\hline
\end{tabular}

in Stickel et al. (1991), we will not consider the forementioned additional sources.

Here we present data for 28 objects observed with the VLA and/or the WSRT; preliminary results on extended emission and polarization properties for a small fraction of these objects were presented in Stanghellini et al. (1997). In this paper we show the images and briefly discuss the results obtained from our observations. In a forthcoming paper we will give a full discussion of the results. Throughout this paper we use $H_{0}=50 \mathrm{~km} \mathrm{~s}^{-1} \mathrm{Mpc}^{-1}$ and $q_{0}=0.5$.

\section{Observation and data reduction}

VLA observations were carried out in 1994 and 1995 at $1.36,1.66,4.8 \mathrm{GHz}$ in $\mathrm{A}, \mathrm{B}$, and $\mathrm{D}$ configurations. WSRT observations took place in Feb. 1994 at 1.4 GHz. In Table 1 we report the journal of these observations.

\subsection{The VLA data}

The VLA data reduction has been performed using the Astronomical Image Processing System (AIPS) developed at the National Radio Astronomy Observatory (NRAO). After a standard calibration we performed several iterations of imaging and phase self-calibration and one final iteration of phase and amplitude self-calibration.

We observed with the VLA in A and B configuration in order to have good sensitivity to radio emitting regions with angular sizes up to 120 arcsec; the high resolution provided by the A-array data allowed a good measure of the core emission and therefore we managed to have an accurate determination of the extended emission flux density. We also planned to combine the data at 1.36 and 1.66 $\mathrm{GHz}$ to improve the $u v$-coverage and increase the sensitivity, but the latter frequency was generally affected by Radio Frequency Interferences (RFI) and we could not pursue our goal. Short B array observations were carried out at $5 \mathrm{GHz}$ to study and compare the arcsecond scale structure detected in the $L$ band. Finally, a few sources were observed in the D array to search for arcminute scale emission (like in $1807+698$ ).

At $1.36 \mathrm{GHz}$ the images were obtained with a multifield clean to remove the contribution of strong sources in the field. The rms noise in the final images is typically in the range $0.07-0.18 \mathrm{mJy} /$ beam, close to the expected thermal noise, with the exception of the sources with declination lower than $-20^{\circ}$. The dynamic range (peak/noise) is between 1000-9000, with typical values around 5000 . The images at $1.66 \mathrm{GHz}$ have been only used to verify the results obtained at $1.36 \mathrm{GHz}$.

We found it useful to combine $L$ band data taken with different configurations only for a few sources in order to increase the sensitivity to the extended emission while maintaining a good resolution. Given that the flux density of the unresolved core may vary significantly between the epochs of the observations in the $\mathrm{A}$ and $\mathrm{B}$ configurations, we had to normalize the core flux density before combining the different data sets of the same source.

From the A and B array observations we obtained two subsets, each containing only the visibilities with common $u v$-coverage. Then we produced an image for each of these subsets and determined the peak flux densities. We subtracted from the original data set with the strongest core a pointlike component with intensity corresponding to the difference between the peak fluxes. Finally we combined the two complete data sets. Only for $1807+698$ we also added the data in the $\mathrm{D}$ configuration, using the same technique.

\subsection{The WSRT data}

The WSRT observations were carried out to search for arcminute scale extended emission (like for VLA D-Array data).

The total observing time for the observations at the WSRT was 24 hours. Each source was observed for typically five 15-min snapshots, inclusive of slewing times, in a range of $\mathrm{HA}$ in order to obtain an acceptable $u v$ coverage. The typical resolution was about $13^{\prime \prime} \times 13^{\prime \prime} \cos$ $\delta$ and the rms noise in the image plane was between 0.15 and $0.3 \mathrm{mJy} /$ beam. The data reduction has been done using the NEWSTAR package that allows redundancy self-calibration. The flux density scale has been referred to Baars et al. (1977) by means of the observations of $3 \mathrm{C} 286$, whose flux density was assumed to be $14.77 \mathrm{Jy}$ at $1.40 \mathrm{GHz}$.

The NEWSTAR package allows for the removal of unresolved components (i.e. components with size much smaller than the observing beam) in the imaging process. This has been used to remove the nucleus of each BL Lac 
object in order to estimate the total flux density of the extended emission.

\subsection{Additional images}

We also searched for images of the 1 Jy BL Lac sources in the NRAO VLA Sky Survey (NVSS, Condon et al. 1998) and in the FIRST (Becker et al. 1995) survey. Most sources appeared pointlike, except $1514-241$ on the NVSS and $0828+493$ and $1418+546$ on the FIRST. These three images are presented here along with the images obtained from our data, since they show more details than those available in the literature.

\section{Results}

The VLA and WSRT observations detected extended emission around about $85 \%$ of the observed objects; for about $50 \%$ of them the flux density of the extended emission we measured is a factor 1.3-3 higher than previously derived from images with lower dynamic range (Antonucci \& Ulvestad 1985; Antonucci et al. 1986; Murphy et al. 1993; Kollgaard et al. 1992; Kollgaard et al. 1996). The WSRT and VLA D-array data were effective on the detection of arc-minute scale structures, but only in a few cases we observed significant emission at this scale above a surface brightness of $\sim 1 \mathrm{mJy} /$ beam.

The images are shown in Fig. 1 through 34 and the image parameters are reported in Table 2. More information on this table is given in the caption. For all the images the contour levels are $-3,3,6,12,25,50,100,200,400,800$, 1500,3000 times the image rms.

The relevant result for our study is the radio luminosity of the extended structure, but we have derived other important physical parameters from the images. The flux density of the extended emission has been determined by subtracting the peak from the total flux density, using the most suitable image, i.e. the image with enough resolution to isolate the core, but still containing all the extended emission. To calculate the luminosity we used the luminosity distance obtained from the redshift. For sources having only a lower limit to the redshift we used this lower value.

Table 3 contains the flux density and luminosity of the extended emission, the largest angular and linear size, the core prominence parameter $R$ and the "sidedness" of the extended emission of the source: 1 if one-sided, 2 if two-sided, $P$ for a pointlike source. The luminosities were $\mathrm{K}$-corrected by the factor $(1+z)^{\alpha_{\text {ext }}-1}$, where $\alpha_{\text {ext }}$ is the spectral index of the extended structure $\left(S \propto \nu^{-\alpha}\right)$ and we have used $\alpha_{\text {ext }}=0.8$. In Table 3 we also report the flux densities of the extended emission, as found in the literature, of the remaining 6 sources from the 1 Jy sample and of a few sources also observed by us, whenever the measure found in the literature had a better resolution allowing a more accurate subtraction of the core emission. When necessary, the measures have been scaled to $1.36 \mathrm{GHz}$.

The largest angular and linear sizes have been calculated only for the sources showing a well defined extended structure. For the one-sided objects we give the largest angular distance from the core.

The core prominence parameter, $R$, is defined as the ratio between the core and the extended emission flux densities, multiplied by $(1+z)^{-\alpha_{\text {ext }}}$, to apply the K-correction, assuming $\alpha_{\text {core }}=0$ for the core spectral index.

In the WSRT images, the cross marks the position of the subtracted core component. For $0454+844,0814+425$, $0820+225,0851+202,1749+701,1823+568$, and 2254 +074 , observed with the WSRT, and 0823+033 (VLA, $\mathrm{D}$ configuration) we could not evaluate the contribution of the core and the extended components, due to the relatively low resolution of these arrays; for $0716+714$ our measure of the extended emission flux density is lower than that found by Antonucci et al. (1986) due to the low resolution of our data. No image is shown for these sources.

We give now a brief description for each source that showed extended structure and a comparison with previous observations.

0048-097: Figure 1 shows the $\mathrm{A}+\mathrm{B}$ configuration image. The core emission is located at the center of a diffuse structure and a bright knot (hot-spot?) is seen at the southern edge of the radio source. The $4.88 \mathrm{GHz}$ image (Fig. 2) from the B array does not show all the extended structure seen at lower frequency. The image at $1.46 \mathrm{GHz}$ published by Wardle et al. (1984) only shows the core and a hint of the southern knot. We find a flux density of $176 \mathrm{mJy}$ in the extended emission to be compared to the $95 \mathrm{mJy}$ reported by Antonucci \& Ulvestad (1985) based on the image of Wardle et al. (1984). 0048-097 appears only marginally resolved in our D array data.

0118-272: the radio image from the A array (1.36 GHz in Fig. 3) shows a halo around the core that contributes significantly to the total luminosity, while the $\mathrm{B}$ configuration image (not shown) displays only a marginally resolved structure. In the image at $4.88 \mathrm{GHz}$ (Fig. 4) there is a hint of a jet extending towards SE. Previous observations of this source are reported by Perley (1982). Our higher dynamic range allows to image the extended emission to larger distances than in Perley (1982); the diffuse emission in our image extends to 18 arcseconds.

0138-097: in the combined A and B array data sets at $1.36 \mathrm{GHz}$ (Fig. 5) we see a weak elongated structure 
Table 2. Image parameters. We do not present images for the sources marked with an asterisk because they are unresolved or because the extended structure in our images does not improve the information in the literature. Column [1] IAU name, Col. [2] array (A, B and D, are for the VLA configurations, W is for the WSRT). "a" and "b" means that the image is from the FIRST or NVSS, respectively, Col. [3] frequency of the observations, Col. [4] beam major and minor axes, Col. [5] position angle of the restoring beam, Col. [6] rms noise on the image plane, Col. [7] peak flux density on the image. When the core has been subtracted, the flux density not restored in the image is shown in parentheses

\begin{tabular}{|c|c|c|c|c|c|c|}
\hline$[1]$ & {$[2]$} & {$[3]$} & {$[4]$} & {$[5]$} & {$[6]$} & {$[7]$} \\
\hline name & array & $\begin{array}{c}\nu \\
(\mathrm{GHz})\end{array}$ & $\begin{array}{c}\text { beam } \\
(\operatorname{arcsec})\end{array}$ & $\begin{array}{l}\mathrm{PA} \\
\left({ }^{\circ}\right)\end{array}$ & $\begin{array}{r}\mathrm{rms} \\
(\mathrm{mJy} / \mathrm{beam})\end{array}$ & $\begin{array}{l}\text { peak } \\
(\mathrm{mJy} / \text { beam })\end{array}$ \\
\hline \multirow[t]{2}{*}{$0048-097$} & $A+B$ & 1.36 & $2.4 \times 1.1$ & 46 & 0.08 & 511 \\
\hline & B & 4.88 & $2.2 \times 1.9$ & -60 & 0.15 & 982 \\
\hline \multirow[t]{2}{*}{$0118-272$} & $\mathrm{~A}$ & 1.36 & $3.0 \times 1.2$ & -21 & 0.10 & 742 \\
\hline & $\mathrm{B}$ & 4.88 & $5.3 \times 1.4$ & 36 & 0.15 & 663 \\
\hline \multirow[t]{2}{*}{$0138-097$} & $\mathrm{~A}+\mathrm{B}$ & 1.36 & $2.3 \times 1.5$ & 42 & 0.07 & 541 \\
\hline & B & 4.88 & $4.3 \times 1.7$ & -53 & 0.15 & 695 \\
\hline \multirow[t]{2}{*}{$0426-380$} & $\mathrm{~A}$ & 1.36 & $4.4 \times 1.0$ & 23 & 0.10 & 624 \\
\hline & $\mathrm{B}$ & 4.88 & $5.3 \times 1.4$ & 15 & 0.15 & 1371 \\
\hline $0454+844 *$ & $\mathrm{~W}$ & 1.40 & $9.2 \times 26.8$ & 57 & 0.20 & $1 \quad(310)$ \\
\hline \multirow[t]{2}{*}{$0537-441$} & $\mathrm{~A}$ & 1.36 & $5.3 \times 1.2$ & 1 & 0.50 & 3010 \\
\hline & $\mathrm{B}$ & 4.88 & $7.1 \times 1.3$ & -3 & 1.80 & 5138 \\
\hline $0716+714 *$ & $\mathrm{~W}$ & 1.40 & $17.5 \times 11.0$ & 0 & 0.30 & $83 \quad(280)$ \\
\hline $0814+425 *$ & $\mathrm{~W}$ & 1.40 & $19.5 \times 13.5$ & 22 & 0.25 & 10 (1099) \\
\hline $0820+225 *$ & W & 1.40 & $33.7 \times 13.8$ & 12 & 0.30 & 81 (1988) \\
\hline $0823+033 *$ & $\mathrm{D}$ & 1.36 & $54.0 \times 43.0$ & -52 & 0.15 & 1479 \\
\hline \multirow{2}{*}{$\begin{array}{r}0828+493 \\
*\end{array}$} & $\mathrm{~B}^{\mathrm{a}}$ & 1.39 & $5.4 \times 5.4$ & 0 & 0.13 & 355 \\
\hline & W & 1.39 & $18.3 \times 13.1$ & 52 & 0.3 & $10(288)$ \\
\hline $0851+202 *$ & W & 1.40 & $39.4 \times 12.5$ & 3 & 0.30 & $2(1143)$ \\
\hline \multirow[t]{2}{*}{$0954+658$} & $\mathrm{~A}$ & 1.36 & $1.1 \times 1.0$ & 34 & 0.08 & 597 \\
\hline & $\mathrm{B}$ & 4.88 & $2.8 \times 1.3$ & 49 & 0.15 & 523 \\
\hline $1144-379$ & $\mathrm{~A}$ & 1.36 & $3.6 \times 1.1$ & -9 & 0.5 & $21 \quad(1892)$ \\
\hline \multirow[t]{2}{*}{$1147+245$} & $A+B$ & 1.36 & $1.3 \times 1.3$ & 0 & 0.07 & 796 \\
\hline & B & 4.88 & $2.6 \times 1.9$ & 82 & 0.10 & 845 \\
\hline $1308+326$ & $\mathrm{~B}$ & 1.46 & $4.3 \times 4.3$ & 0 & 0.15 & 859 \\
\hline \multirow{3}{*}{$1418+546$} & $\mathrm{~B}^{\mathrm{a}}$ & 1.40 & $5.4 \times 5.4$ & 0 & 0.15 & 566 \\
\hline & $\mathrm{W}$ & 1.40 & $17.0 \times 12.0$ & 14 & 0.25 & 19 (710) \\
\hline & $\mathrm{D}$ & 1.36 & $68.0 \times 32.0$ & -69 & 0.07 & 815 \\
\hline \multirow[t]{3}{*}{$1514-241$} & $A+B$ & 1.36 & $3.0 \times 2.0$ & 28 & 0.15 & 1606 \\
\hline & B & 4.88 & $3.0 \times 1.5$ & -50 & 0.17 & 2918 \\
\hline & $\mathrm{D}^{\mathbf{b}}$ & 1.40 & $45 \times 45$ & 0 & 0.40 & 1993 \\
\hline \multirow[t]{2}{*}{$1519-273 *$} & $\mathrm{~A}$ & 1.36 & $3.4 \times 1.4$ & -30 & 0.2 & 1690 \\
\hline & $\mathrm{B}$ & 1.36 & $9.3 \times 3.2$ & 33 & 0.10 & 883 \\
\hline \multirow[t]{2}{*}{$1652+398$} & $\mathrm{~B}$ & 1.36 & $3.5 \times 3.0$ & -84 & 0.13 & 1383 \\
\hline & $\mathrm{B}$ & 4.88 & $1.6 \times 1.3$ & -76 & 0.18 & 1320 \\
\hline $1749+701 *$ & $\mathrm{~W}$ & 1.40 & $18.3 \times 11.6$ & -61 & 0.20 & $1(650)$ \\
\hline \multirow[t]{2}{*}{$1803+784$} & $\mathrm{~B}$ & 1.36 & $9.3 \times 4.9$ & -11 & 0.07 & 1761 \\
\hline & $\mathrm{B}$ & 4.88 & $2.5 \times 1.2$ & -13 & 0.30 & 2214 \\
\hline \multirow[t]{3}{*}{$1807+698$} & $\mathrm{~A}$ & 1.36 & $3.2 \times 1.5$ & -49 & 0.15 & 1136 \\
\hline & $\mathrm{A}+\mathrm{B}+\mathrm{D}$ & 1.36 & $7.0 \times 5.5$ & -22 & 0.10 & 1284 \\
\hline & B & 4.88 & $2.5 \times 1.3$ & -9 & 0.15 & 1507 \\
\hline $1823+568 *$ & $\mathrm{~W}$ & 1.40 & $30.0 \times 10.0$ & -53 & 0.30 & $50 \quad(1190)$ \\
\hline $2007+777$ & $\mathrm{~W}$ & 1.40 & $24.0 \times 10.0$ & -47 & 0.25 & 17 (1095) \\
\hline \multirow[t]{2}{*}{$2131-021$} & $\mathrm{~A}$ & 1.36 & $1.6 \times 1.5$ & 1 & 0.20 & 1291 \\
\hline & $\mathrm{B}$ & 4.88 & $1.9 \times 1.4$ & -22 & 0.20 & 1669 \\
\hline \multirow[t]{2}{*}{$2240-260$} & $\mathrm{~A}$ & 1.36 & $2.0 \times 1.0$ & 6 & 0.08 & 813 \\
\hline & $\mathrm{B}$ & 4.88 & $3.7 \times 1.3$ & -29 & 0.20 & 805 \\
\hline $2254+074 *$ & $\mathrm{D}$ & 1.36 & $47.0 \times 42.0$ & 4 & 0.15 & 284 \\
\hline
\end{tabular}




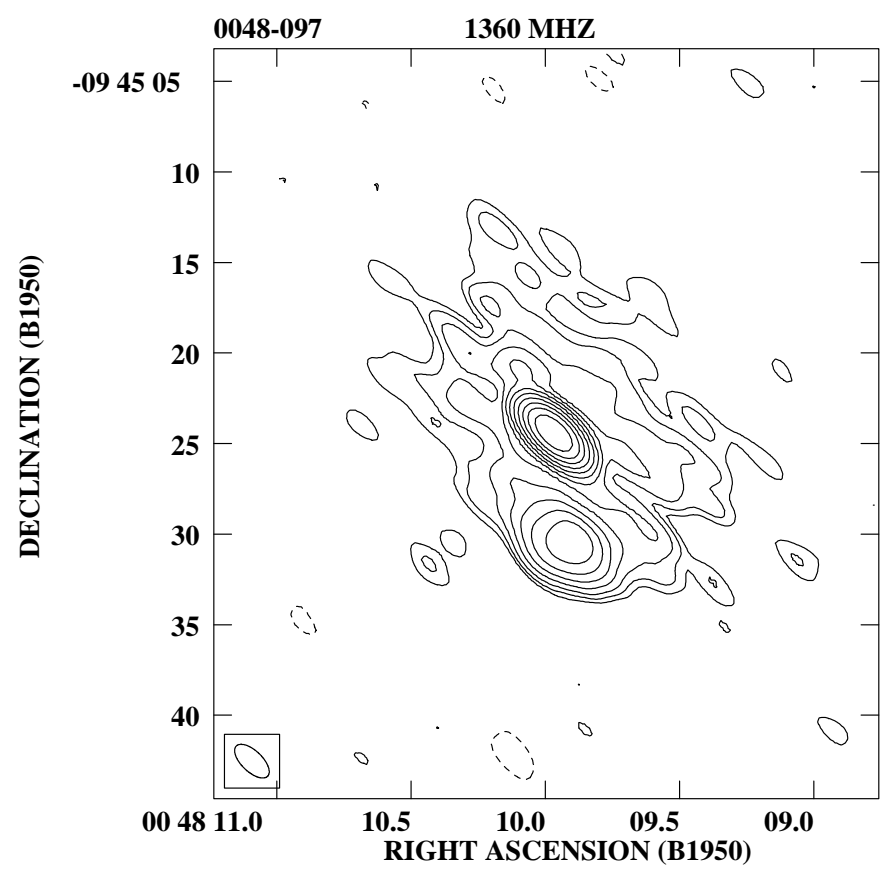

Fig. 1. 0048-097, VLA A+B configuration, 1.36 GHz. The restoring beam is $2.4 \times 1.1$ arcsec in $\mathrm{PA} 46^{\circ}$. The peak flux density is $511 \mathrm{mJy} /$ beam and the rms noise on the image is $0.08 \mathrm{mJy} /$ beam

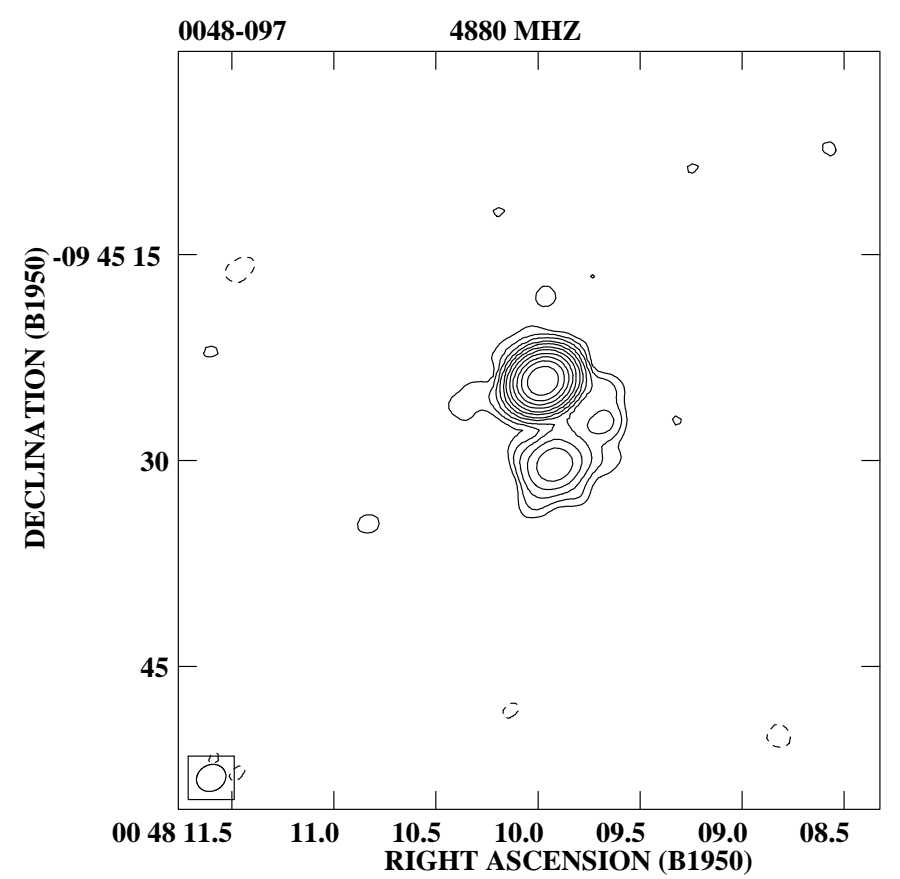

Fig. 2. 0048-097, VLA B configuration, $4.88 \mathrm{GHz}$. The restoring beam is $2.2 \times 1.9$ arcsec in PA $-60^{\circ}$. The peak flux density is $982 \mathrm{mJy} /$ beam and the rms noise on the image is $0.15 \mathrm{mJy} /$ beam

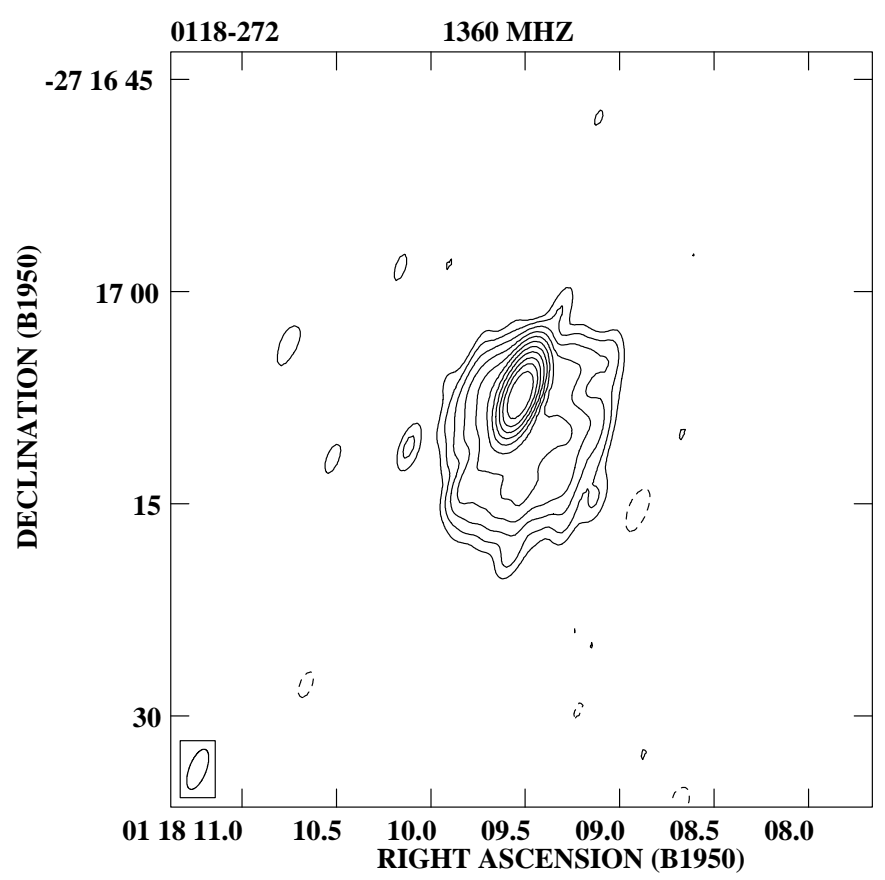

Fig. 3. 0118-272, VLA A configuration, $1.36 \mathrm{GHz}$. The restoring beam is $3.0 \times 1.2 \operatorname{arcsec}$ in PA $-21^{\circ}$. The peak flux density is $742 \mathrm{mJy} / \mathrm{beam}$ and the $\mathrm{rms}$ noise on the image is $0.10 \mathrm{mJy} /$ beam

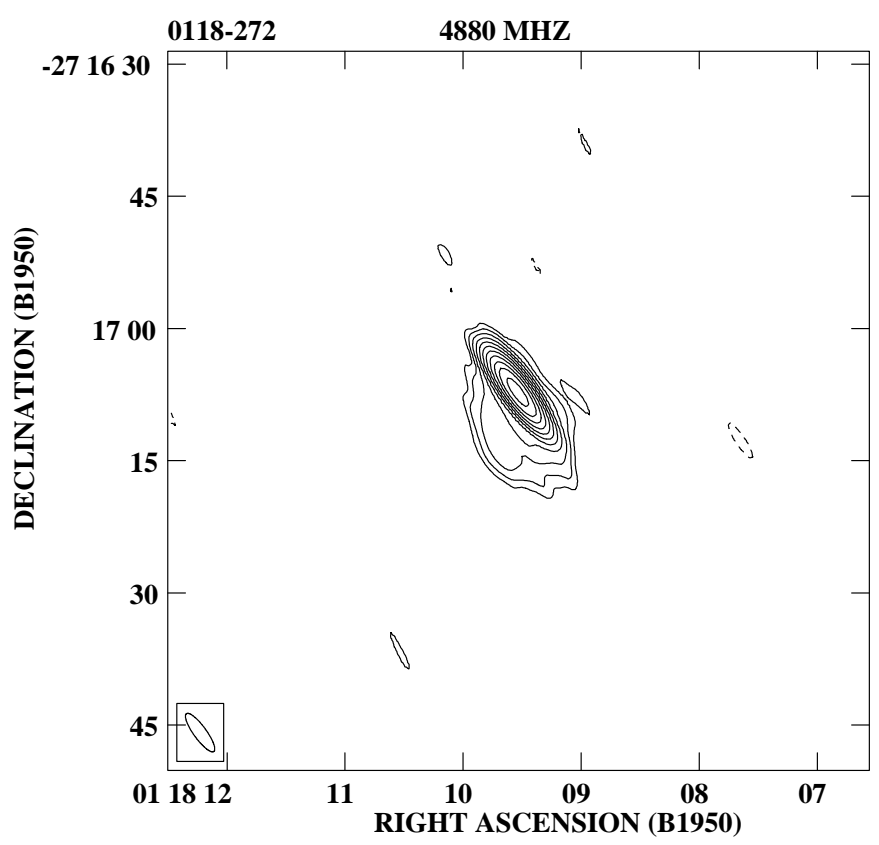

Fig. 4. 0118-272, VLA B configuration, $4.88 \mathrm{GHz}$. The restoring beam is $5.3 \times 1.4$ arcsec in $\mathrm{PA} 36^{\circ}$. The peak flux density is $663 \mathrm{mJy} / \mathrm{beam}$ and the rms noise on the image is $0.15 \mathrm{mJy} /$ beam 


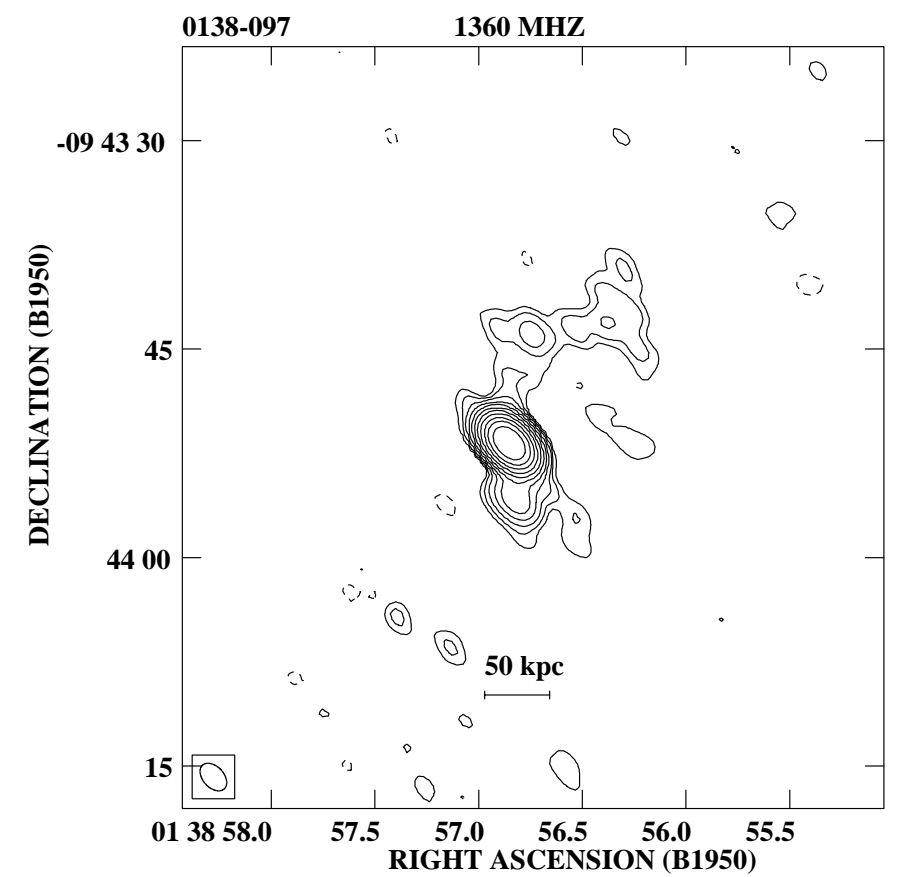

Fig. 5. 0138-097, VLA A+B configuration, $1.36 \mathrm{GHz}$. The restoring beam is $2.3 \times 1.5 \operatorname{arcsec}$ in $\mathrm{PA} 42^{\circ}$. The peak flux density is $541 \mathrm{mJy} /$ beam and the rms noise on the image is $0.07 \mathrm{mJy} /$ beam

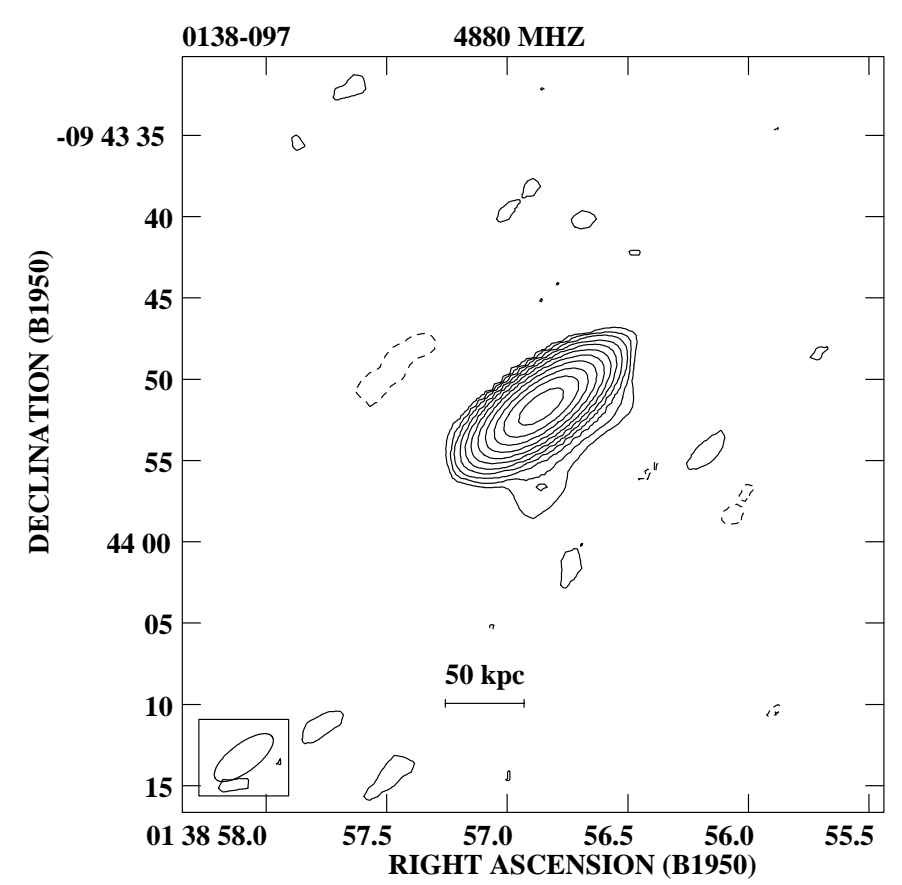

Fig. 6. 0138-097, VLA B configuration, 4.88 GHz. The restoring beam is $4.3 \times 1.7$ arcsec in PA $-53^{\circ}$. The peak flux density is $695 \mathrm{mJy} /$ beam and the rms noise on the image is $0.15 \mathrm{mJy} /$ beam

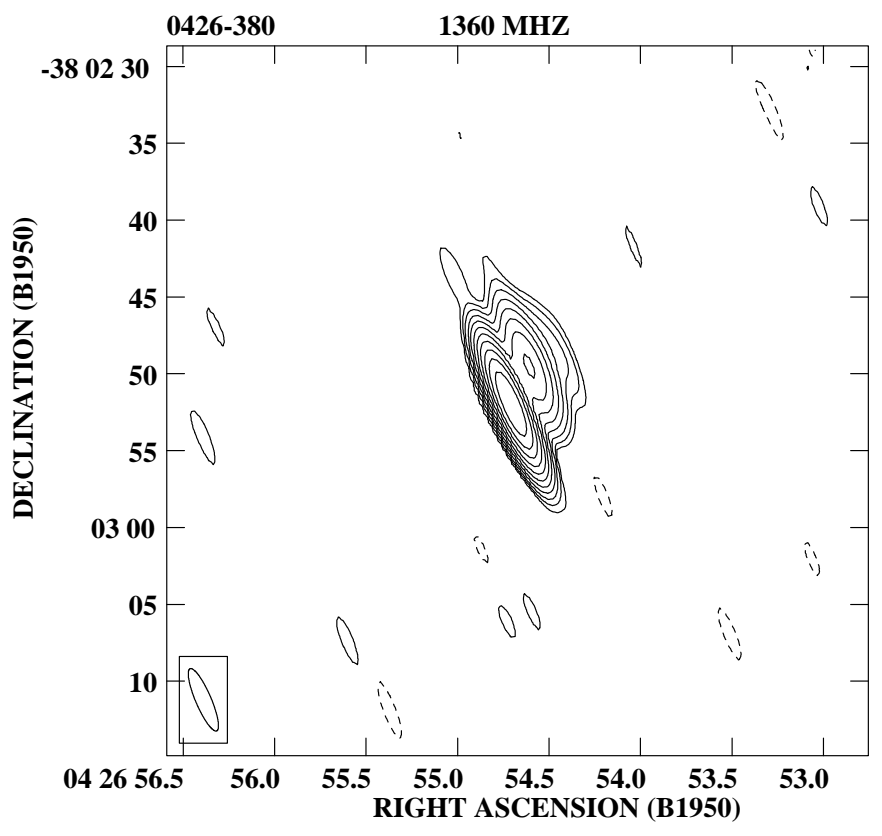

Fig. 7. 0426-380, VLA A configuration, $1.36 \mathrm{GHz}$. The restoring beam is $4.4 \times 1.0$ arcsec in $\mathrm{PA} 23^{\circ}$. The peak flux density is $624 \mathrm{mJy} /$ beam and the rms noise on the image is $0.10 \mathrm{mJy} /$ beam

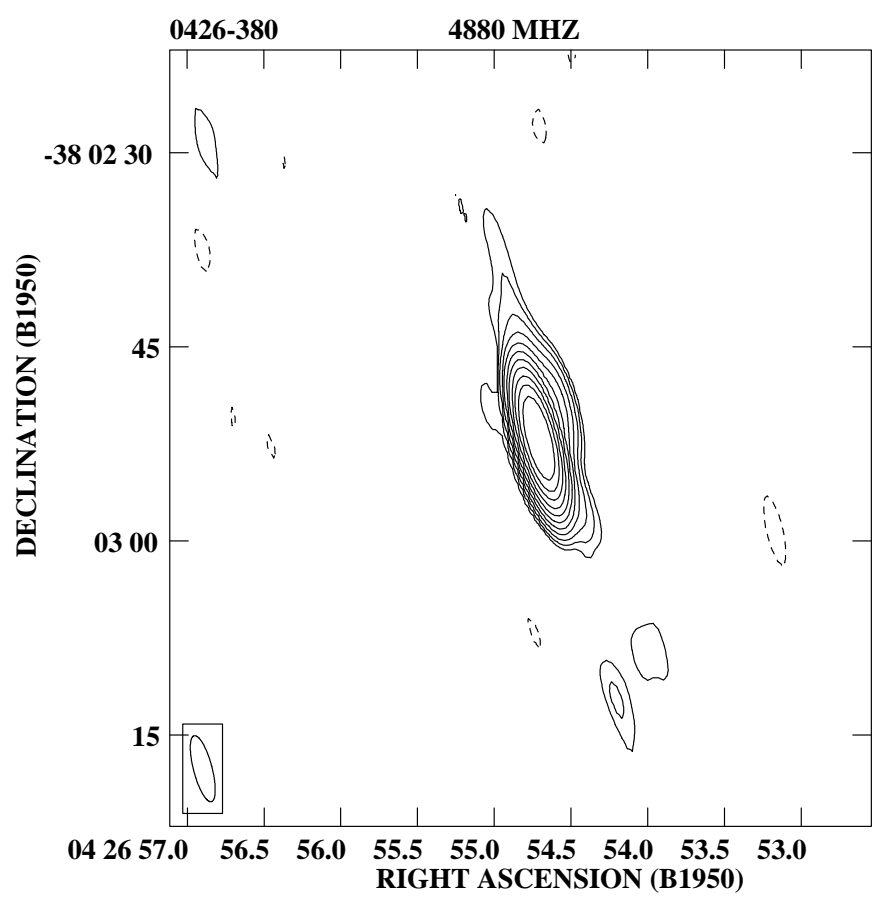

Fig. 8. 0426-380, VLA B configuration, 4.88 GHz. The restoring beam is $5.3 \times 1.4$ arcsec in PA $15^{\circ}$. The peak flux density is $1371 \mathrm{mJy} /$ beam and the rms noise on the image is $0.15 \mathrm{mJy} /$ beam 


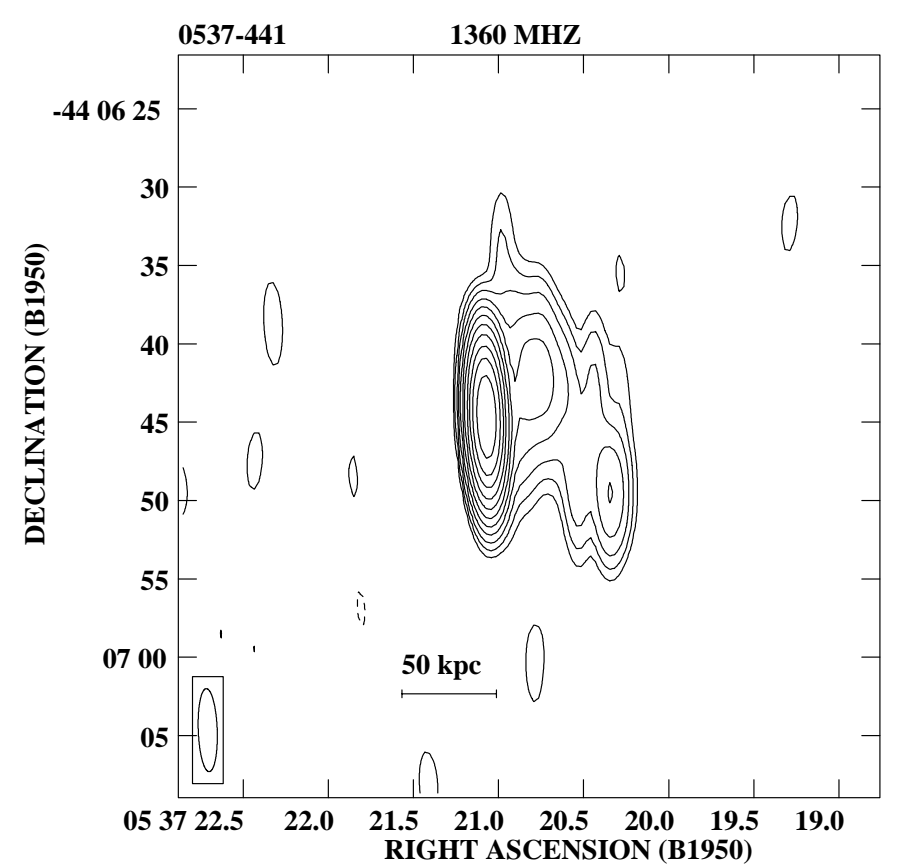

Fig. 9. 0537-441, VLA A configuration, $1.36 \mathrm{GHz}$. The restoring beam is $5.3 \times 1.2 \operatorname{arcsec}$ in PA $1^{\circ}$. The peak flux density is $3010 \mathrm{mJy} /$ beam and the rms noise on the image is $0.50 \mathrm{mJy} /$ beam

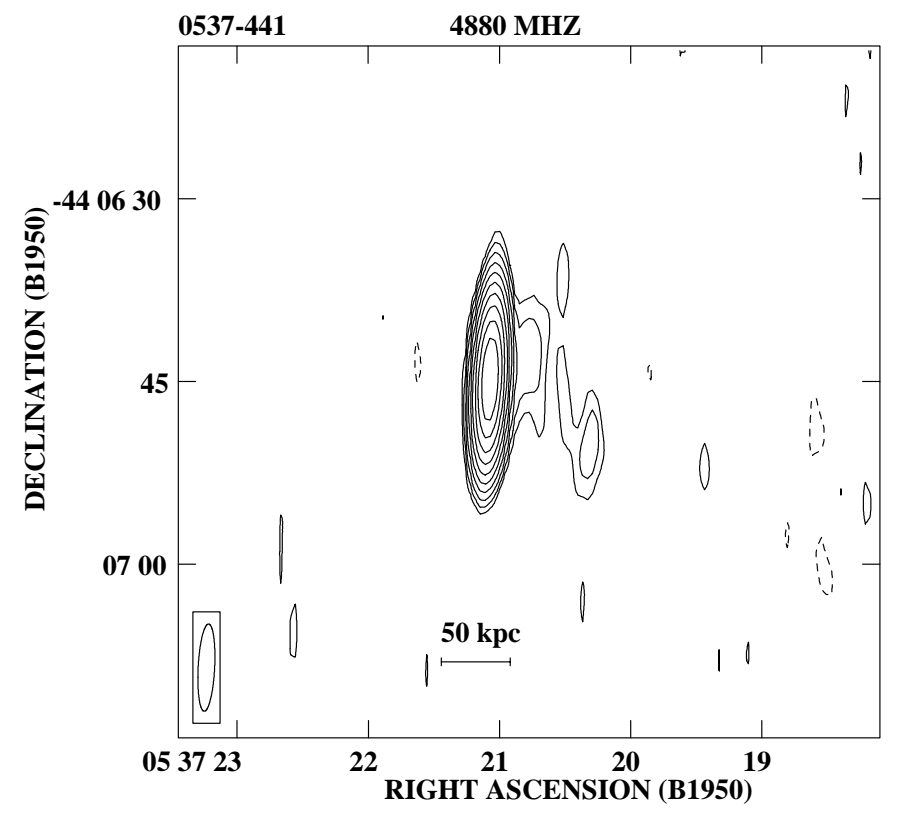

Fig. 10. 0537-441, VLA B configuration, 4.88 GHz. The restoring beam is $7.1 \times 1.3$ arcsec in PA $-3^{\circ}$. The peak flux density is $5138 \mathrm{mJy} /$ beam and the rms noise on the image is $1.80 \mathrm{mJy} /$ beam

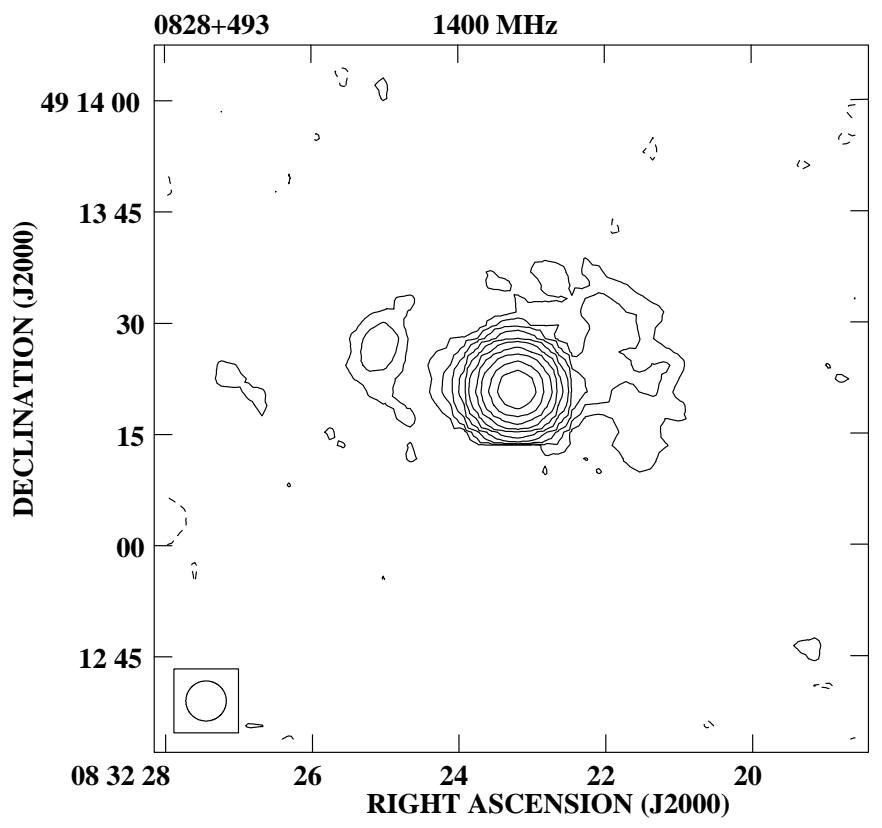

Fig. 11. 0828+493, VLA B configuration, $1.40 \mathrm{GHz}$ (from FIRST, Becker et al. 1995). The restoring beam is $5.4 \times 5.4$ arcsec. The peak flux density is $355 \mathrm{mJy} /$ beam and the $\mathrm{rms}$ noise on the image is $0.13 \mathrm{mJy} /$ beam

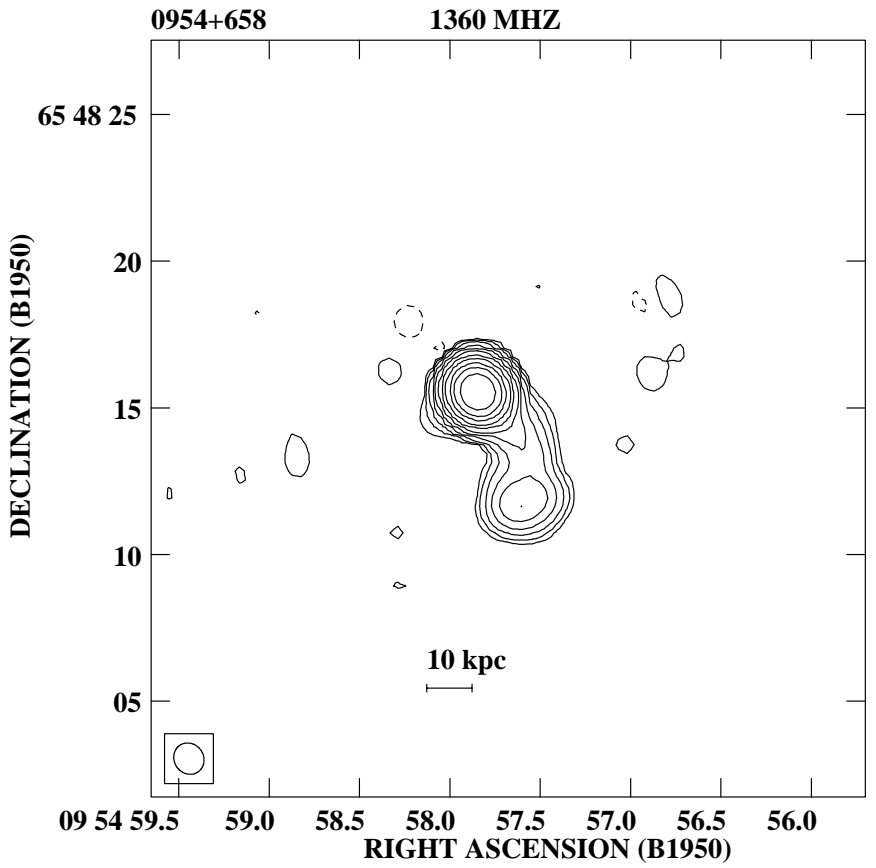

Fig. 12. 0954+658, VLA A configuration, $1.36 \mathrm{GHz}$. The restoring beam is $1.1 \times 1.0$ arcsec in PA $34^{\circ}$. The peak flux density is $597 \mathrm{mJy} /$ beam and the rms noise on the image is $0.08 \mathrm{mJy} /$ beam 


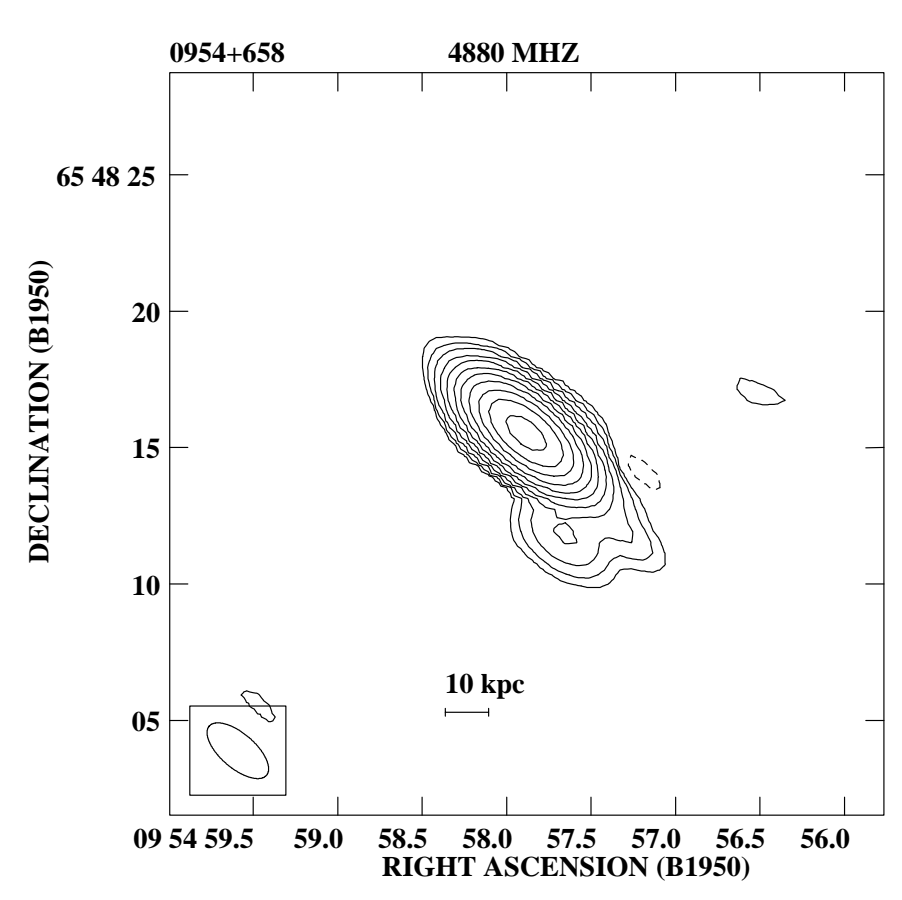

Fig. 13. 0954+658, VLA B configuration, $4.88 \mathrm{GHz}$. The restoring beam is $2.8 \times 1.3$ arcsec in PA $49^{\circ}$. The peak flux density is $523 \mathrm{mJy} /$ beam and the rms noise on the image is $0.15 \mathrm{mJy} /$ beam

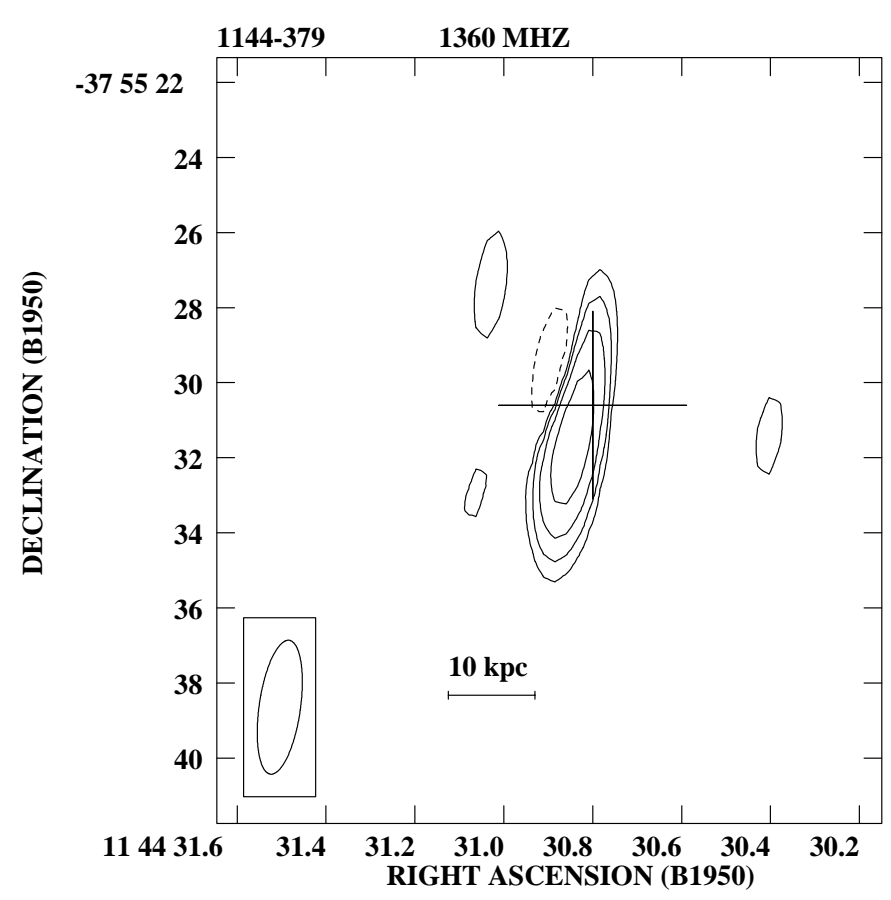

Fig. 14. 1144-379, VLA A configuration, 1.36 GHz. The restoring beam is $3.6 \times 1.1$ arcsec in $\mathrm{PA}-9^{\circ}$. The peak flux density is $21 \mathrm{mJy} /$ beam and the rms noise on the image is $0.5 \mathrm{mJy} /$ beam

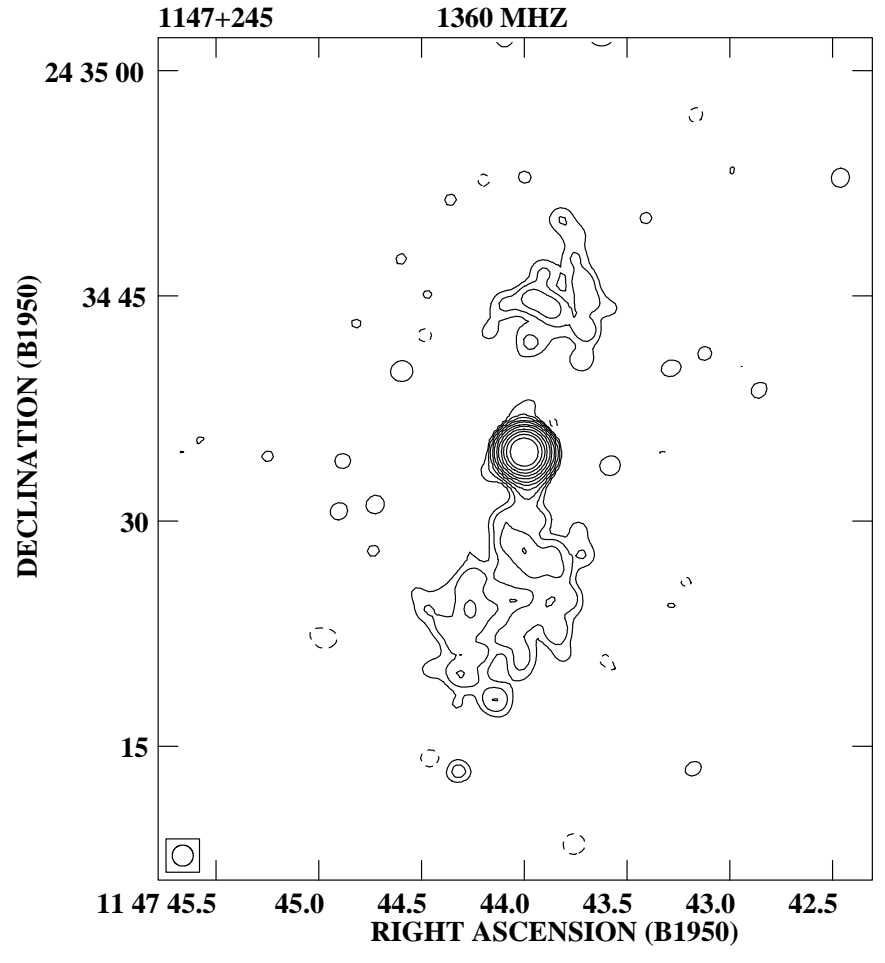

Fig. 15. $1147+245$, VLA A+B configuration, $1.36 \mathrm{GHz}$. The restoring beam is $1.3 \times 1.3$ arcsec. The peak flux density is $796 \mathrm{mJy} /$ beam and the $\mathrm{rms}$ noise on the image is $0.07 \mathrm{mJy} /$ beam

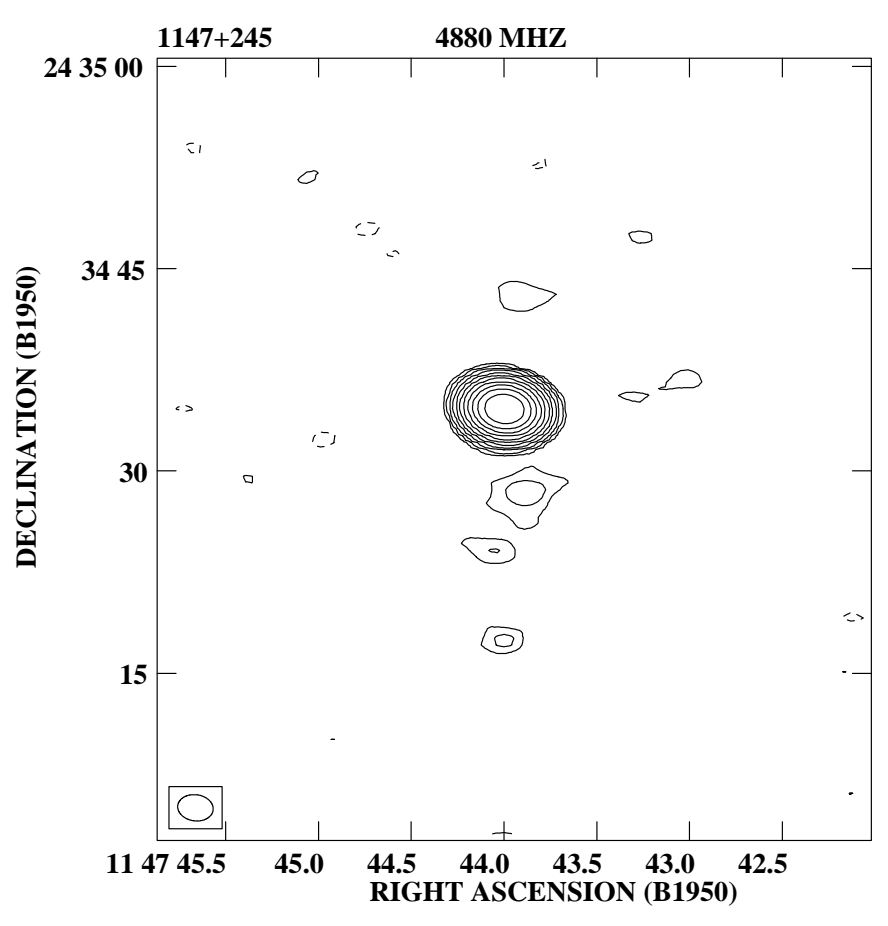

Fig. 16. $1147+245$, VLA B configuration, $4.88 \mathrm{GHz}$. The restoring beam is $2.6 \times 1.9$ arcsec in PA $82^{\circ}$. The peak flux density is $845 \mathrm{mJy} /$ beam and the rms noise on the image is $0.10 \mathrm{mJy} /$ beam 


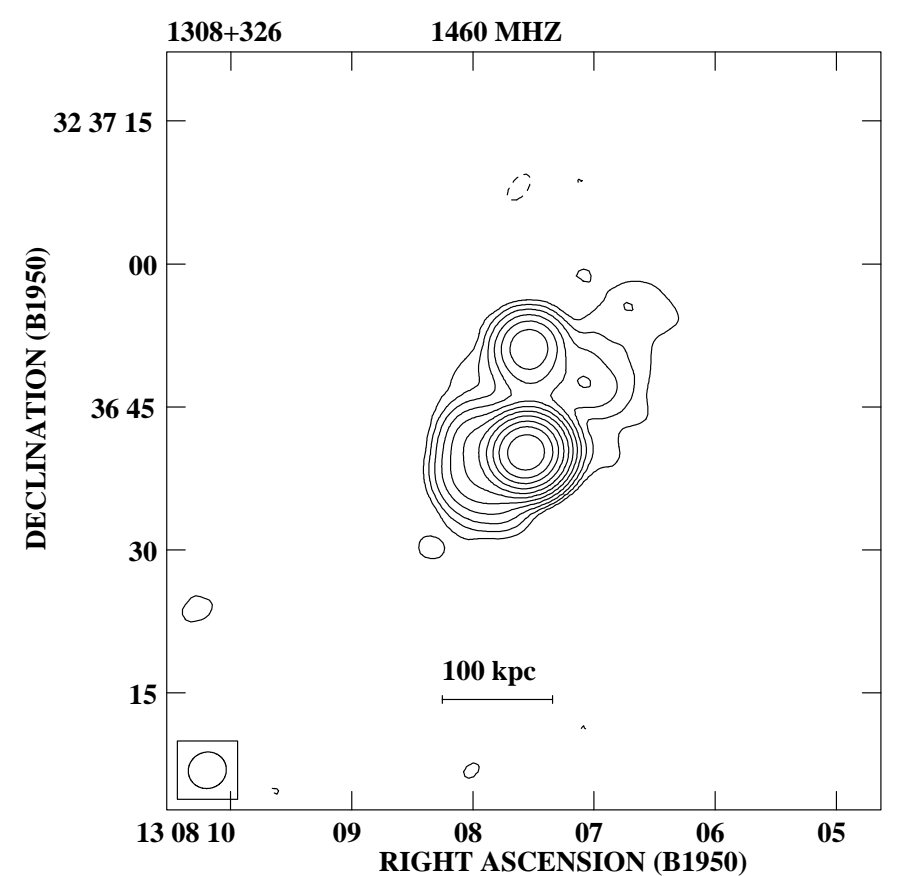

Fig. 17. 1308+326, VLA B configuration, $1.46 \mathrm{GHz}$. The restoring beam is $4.3 \times 4.3$ arcsec. The peak flux density is $859 \mathrm{mJy} /$ beam and the rms noise on the image is $0.15 \mathrm{mJy} /$ beam

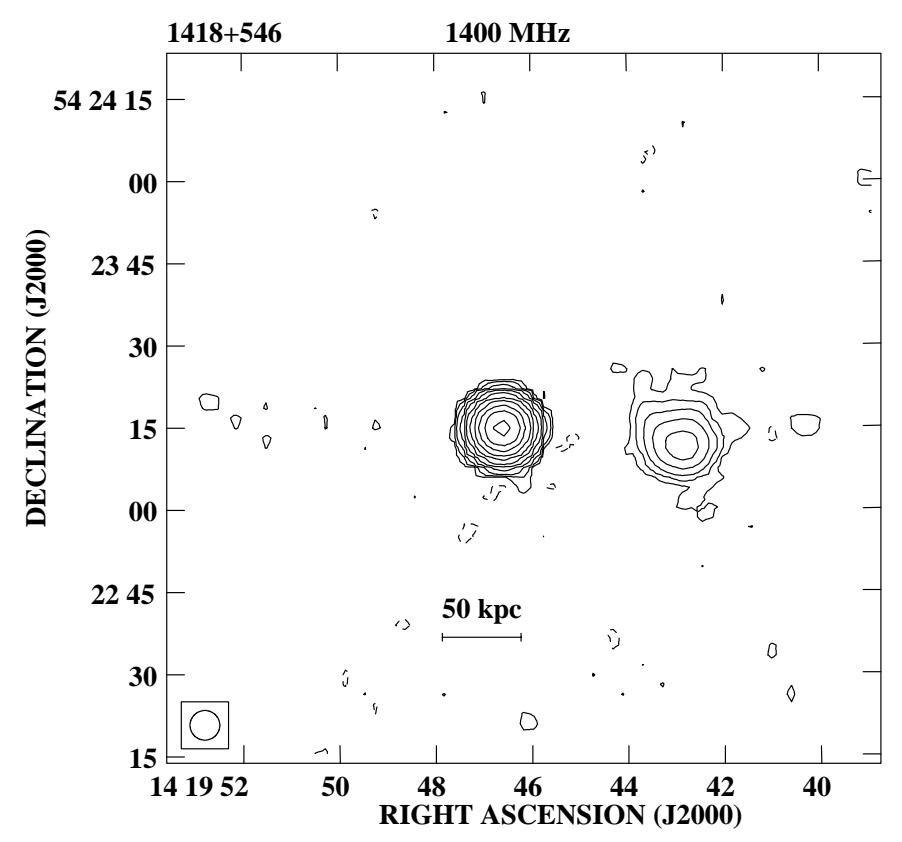

Fig. 18. 1418+546, VLA B configuration, $1.40 \mathrm{GHz}$ (from FIRST, Becker et al. 1995). The restoring beam is $5.4 \times 5.4$ arcsec. The peak flux density is $566 \mathrm{mJy} /$ beam and the rms noise on the image is $0.15 \mathrm{mJy} /$ beam

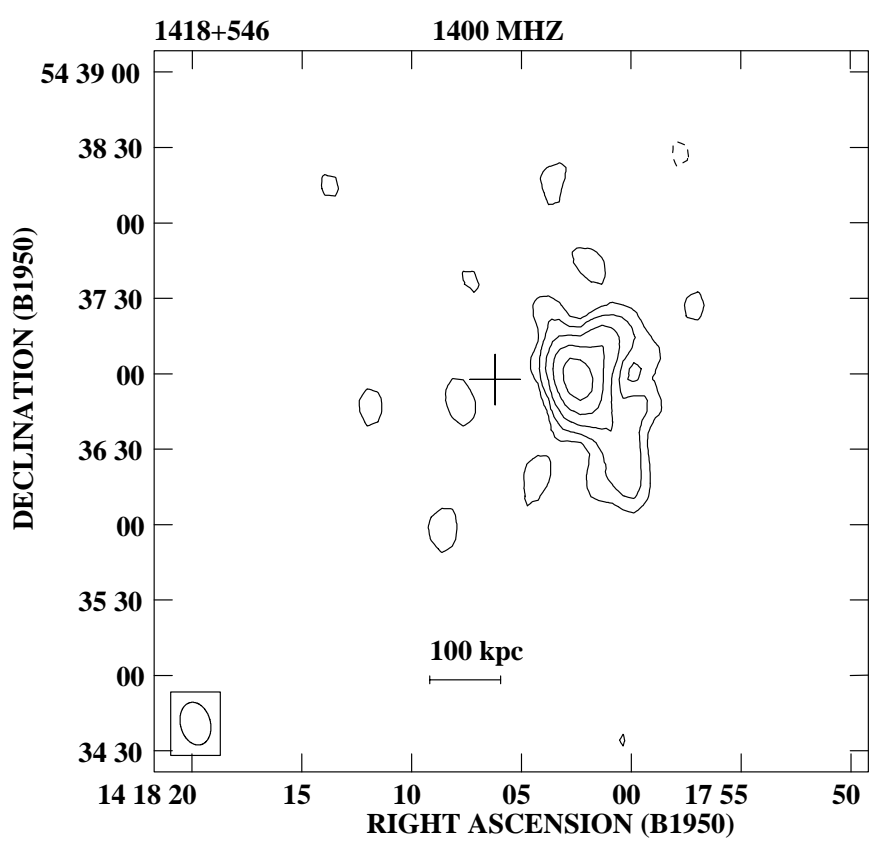

Fig. 19. 1418+546, WSRT, $1.40 \mathrm{GHz}$. The restoring beam is $17.0 \times 12.0 \operatorname{arcsec}$ in PA $14^{\circ}$. The peak flux density is $19 \mathrm{mJy} /$ beam and the rms noise on the image is $0.25 \mathrm{mJy} /$ beam

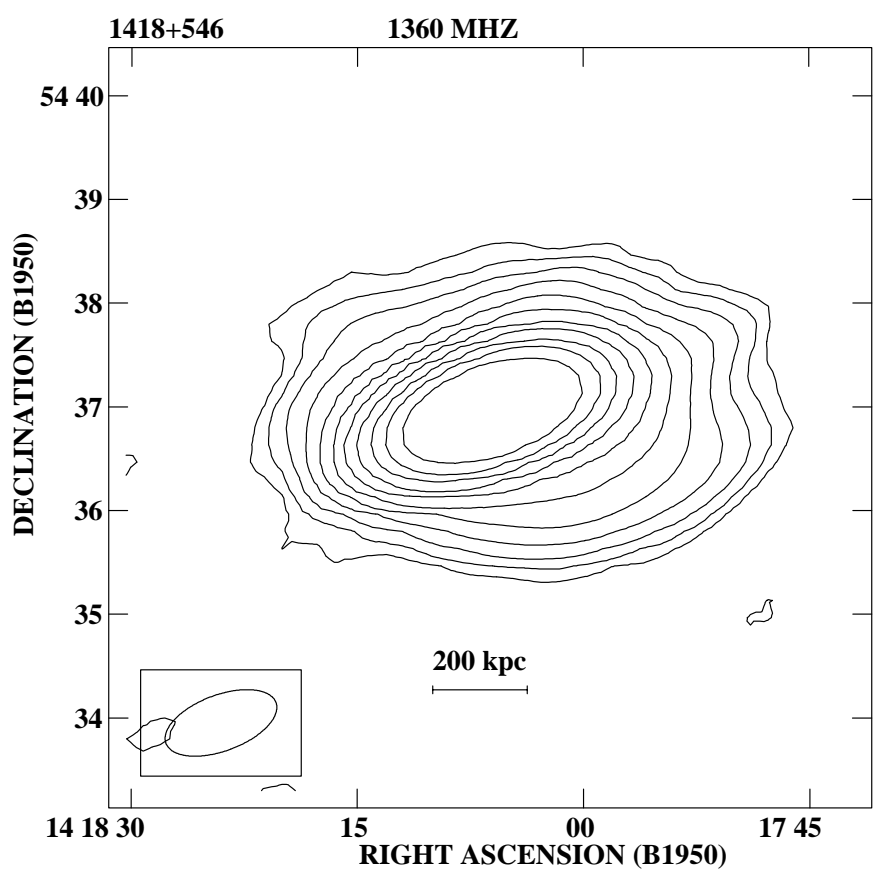

Fig. 20. 1418+546, VLA D configuration, $1.36 \mathrm{GHz}$. The restoring beam is $68.0 \times 32.0$ arcsec in $\mathrm{PA}-69^{\circ}$. The peak flux density is $815 \mathrm{mJy} /$ beam and the rms noise on the image is $0.07 \mathrm{mJy} /$ beam 


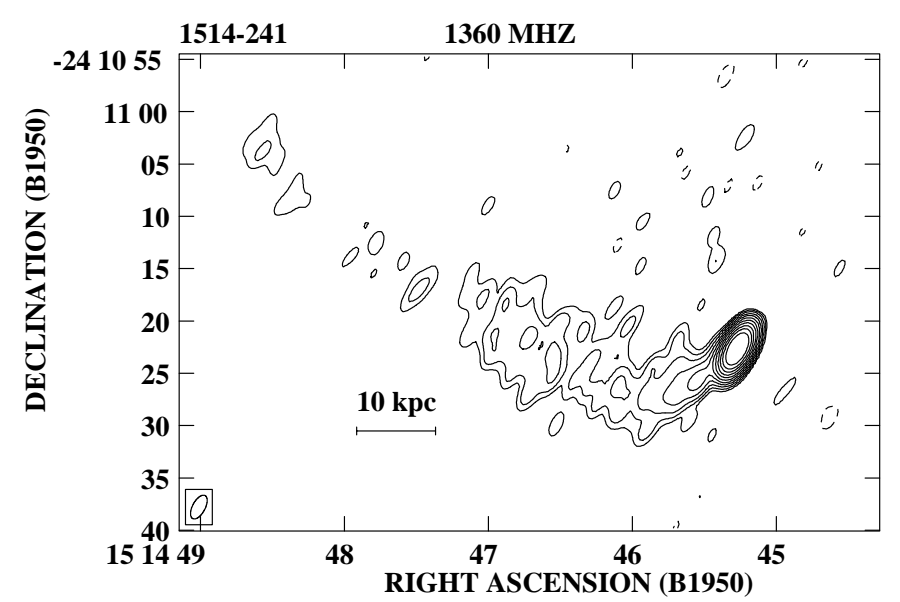

Fig. 21. 1514-241, VLA A+B configuration, 1.36 GHz. The restoring beam is $3.0 \times 2.0$ arcsec in $\mathrm{PA} 28^{\circ}$. The peak flux density is $1625 \mathrm{mJy} /$ beam and the rms noise on the image is $0.15 \mathrm{mJy} /$ beam

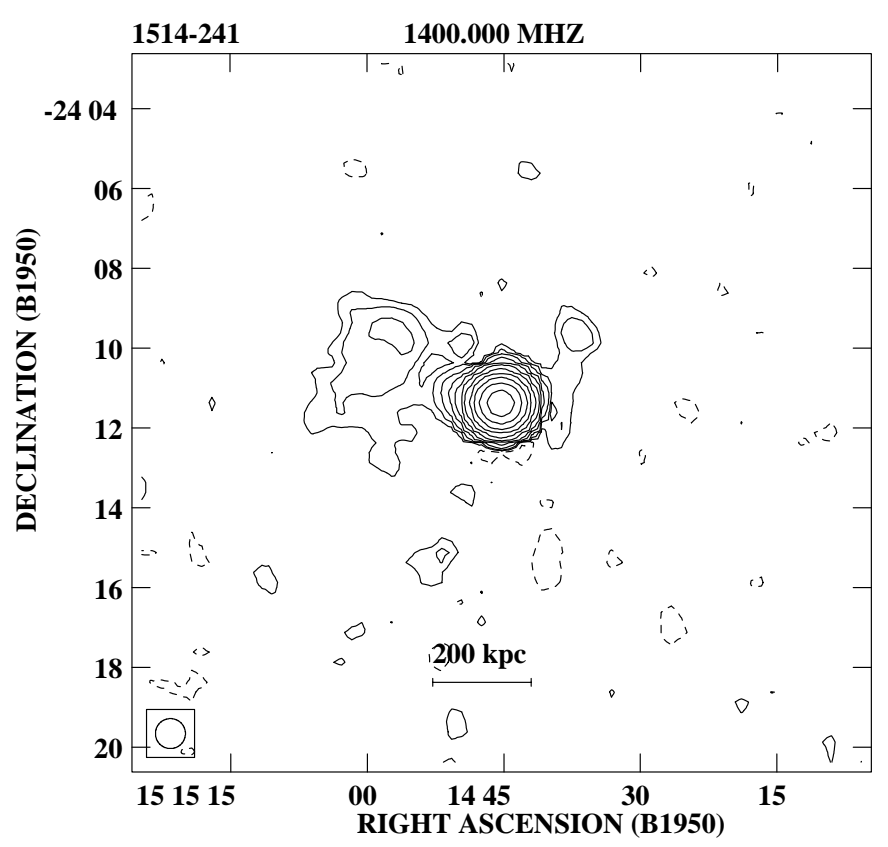

Fig. 23. 1514-241, VLA D configuration, $1.40 \mathrm{GHz}$ (from NVSS, Condon et al. 1998). The restoring beam is $45.0 \times$ 45.0 arcsec. The peak flux density is $1993 \mathrm{mJy} /$ beam and the rms noise on the image is $0.40 \mathrm{mJy} /$ beam

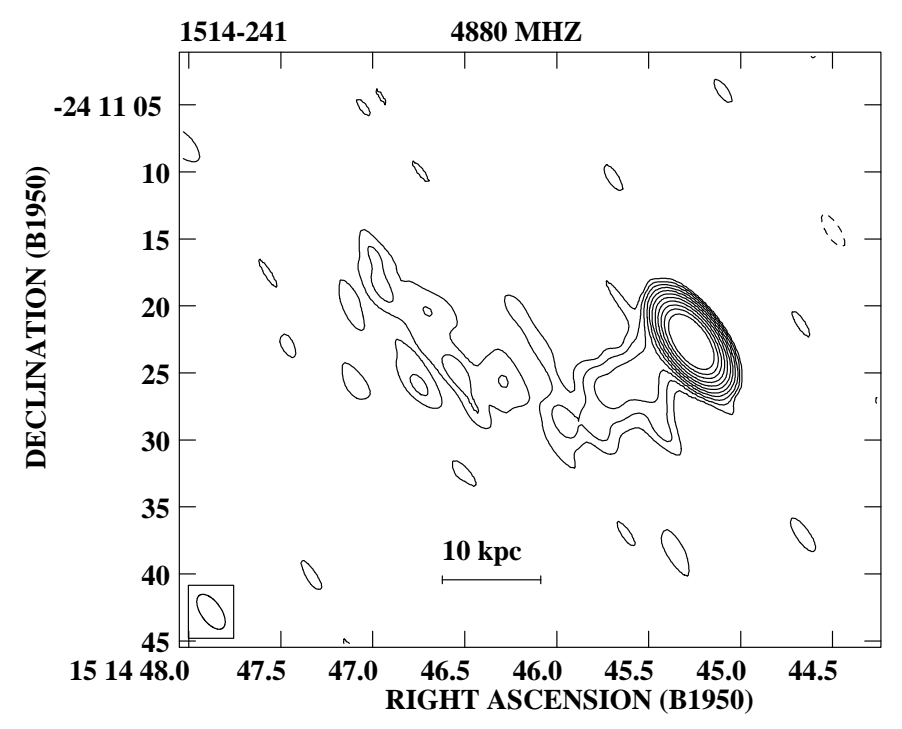

Fig. 22. 1514-241, VLA B configuration, $4.88 \mathrm{GHz}$. The restoring beam is $3.0 \times 1.5$ arcsec in $\mathrm{PA}-50^{\circ}$. The peak flux density is $2918 \mathrm{mJy} /$ beam and the rms noise on the image is $0.17 \mathrm{mJy} /$ beam

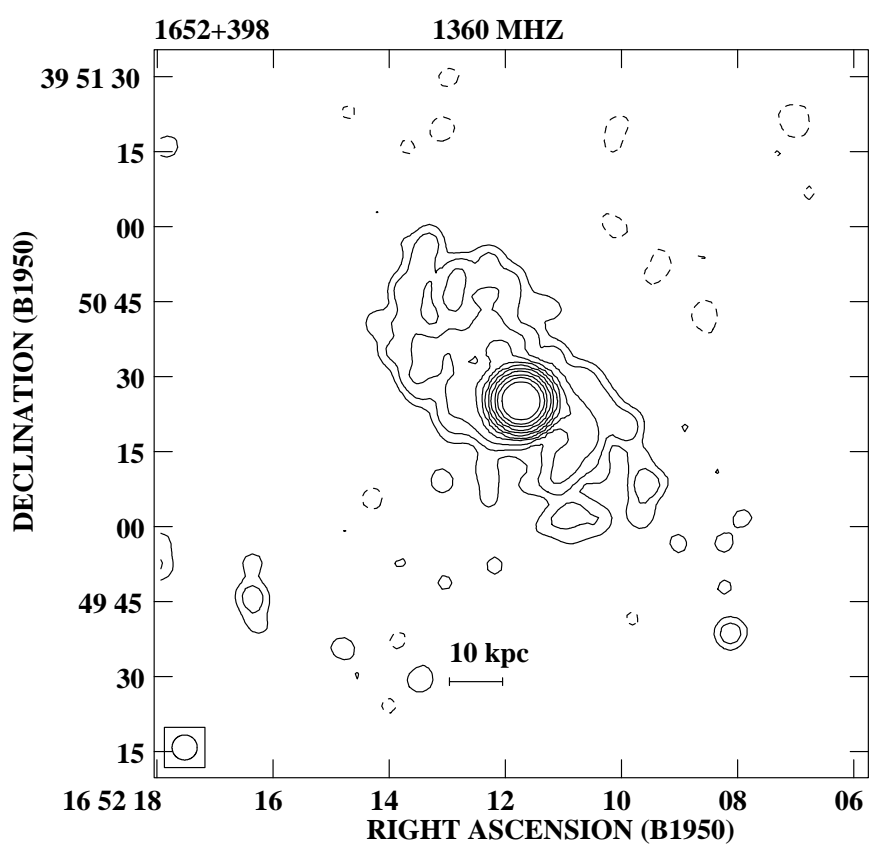

Fig. 24. 1652+398, VLA B configuration, $1.36 \mathrm{GHz}$. The restoring beam is $3.5 \times 3.0 \operatorname{arcsec}$ in $\mathrm{PA}-84^{\circ}$. The peak flux density is $1383 \mathrm{mJy} /$ beam and the rms noise on the image is $0.13 \mathrm{mJy} /$ beam 


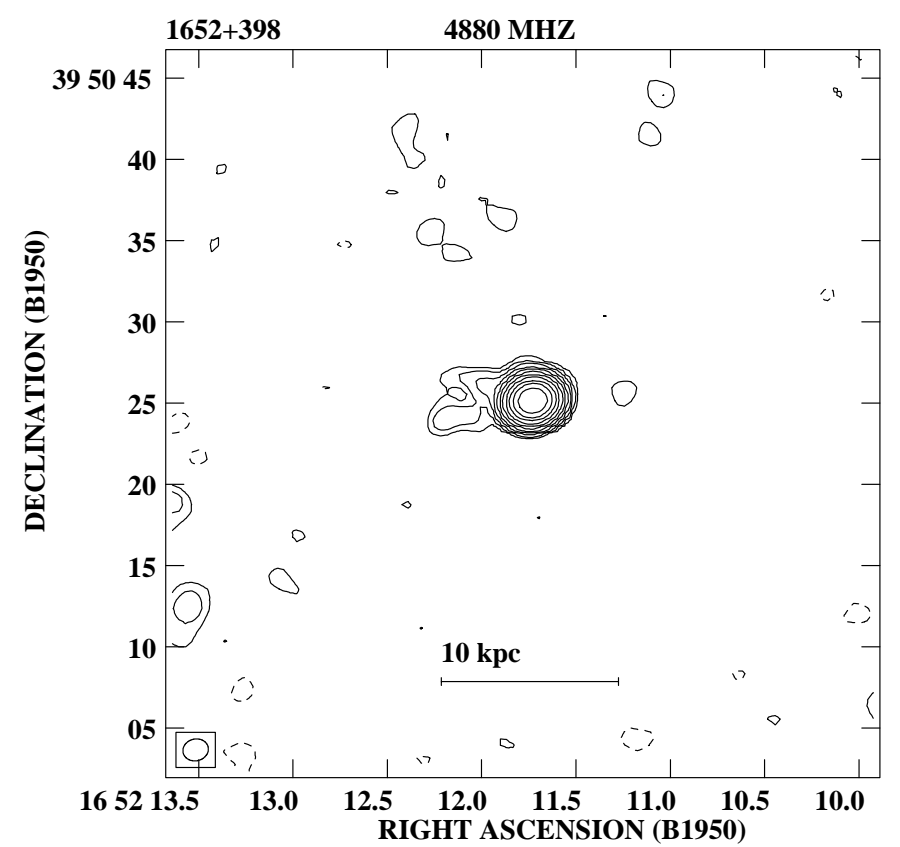

Fig. 25. 1652+398, VLA B configuration, $4.88 \mathrm{GHz}$. The restoring beam is $1.6 \times 1.3$ arcsec in $\mathrm{PA}-76^{\circ}$. The peak flux density is $1320 \mathrm{mJy} /$ beam and the rms noise on the image is $0.18 \mathrm{mJy} /$ beam

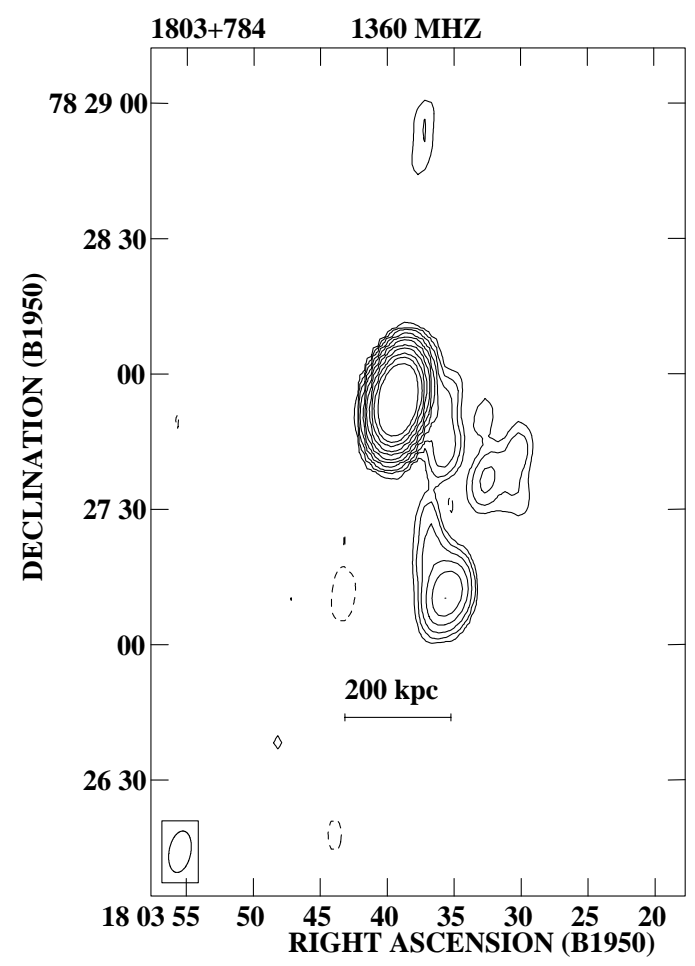

Fig. 26. 1803+784, VLA B configuration, $1.36 \mathrm{GHz}$. The restoring beam is $9.3 \times 4.9 \operatorname{arcsec}$ in $\mathrm{PA}-11^{\circ}$. The peak flux density is $1761 \mathrm{mJy} /$ beam and the rms noise on the image is $0.07 \mathrm{mJy} /$ beam

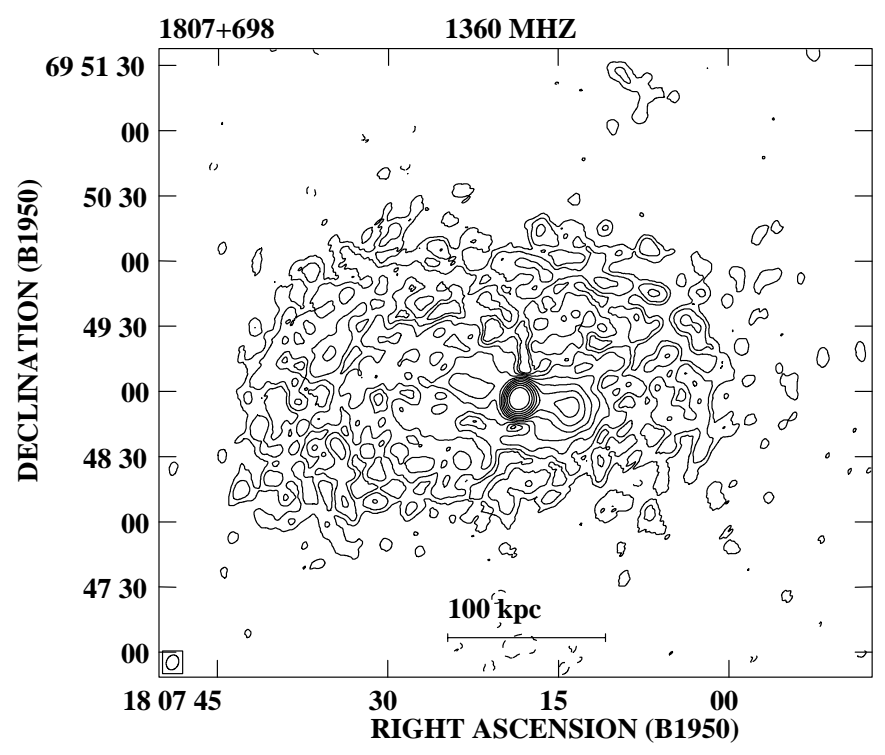

Fig. 27. 1807+698, VLA A+B+D configuration, $1.36 \mathrm{GHz}$. The restoring beam is $7.0 \times 5.5 \operatorname{arcsec}$ in $\mathrm{PA}-22^{\circ}$. The peak flux density is $1284 \mathrm{mJy} /$ beam and the rms noise on the image is $0.10 \mathrm{mJy} /$ beam

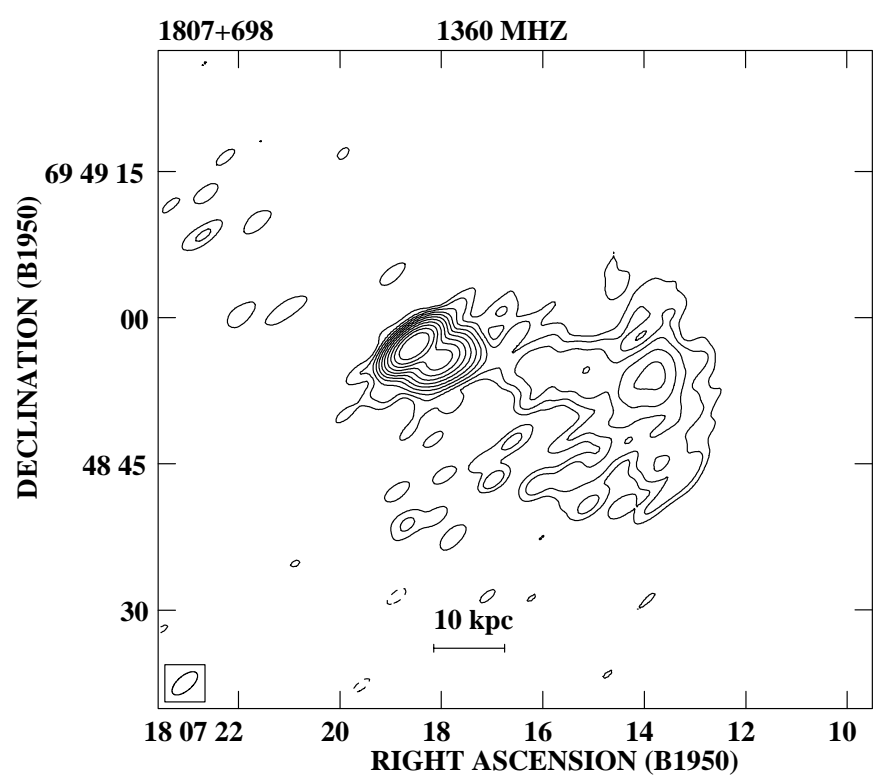

Fig. 28. 1807+698, VLA A configuration, $1.36 \mathrm{GHz}$. The restoring beam is $3.2 \times 1.5$ arcsec in PA $-49^{\circ}$. The peak flux density is $1136 \mathrm{mJy} /$ beam and the rms noise on the image is $0.15 \mathrm{mJy} /$ beam 


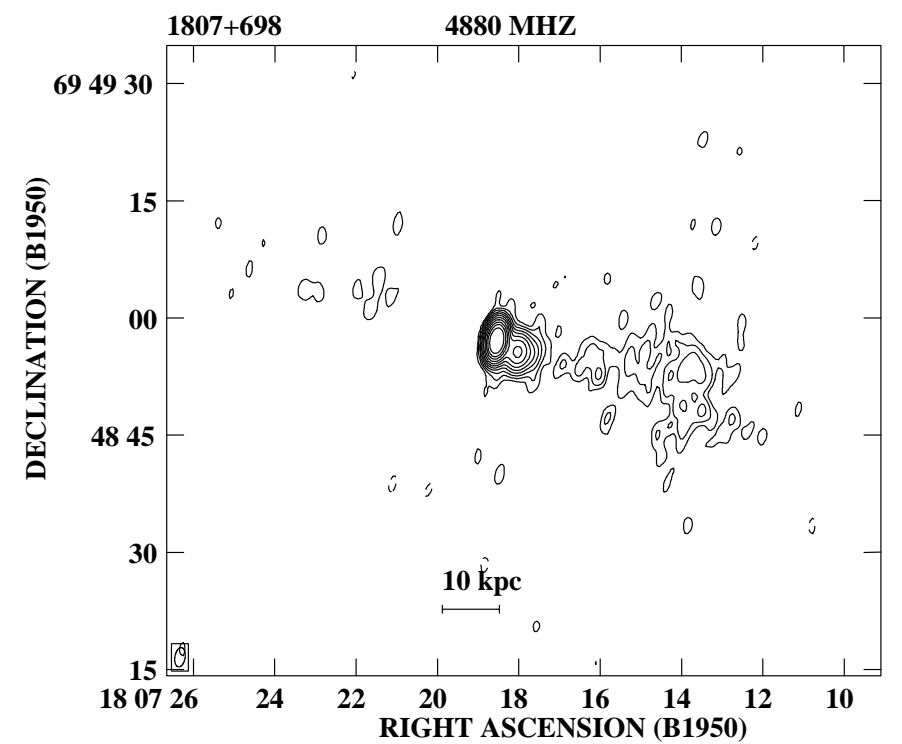

Fig. 29. $1807+698$, VLA B configuration, $4.88 \mathrm{GHz}$. The restoring beam is $2.5 \times 1.3 \operatorname{arcsec}$ in $\mathrm{PA}-9^{\circ}$. The peak flux density is $1507 \mathrm{mJy} /$ beam and the rms noise on the image is $0.15 \mathrm{mJy} /$ beam

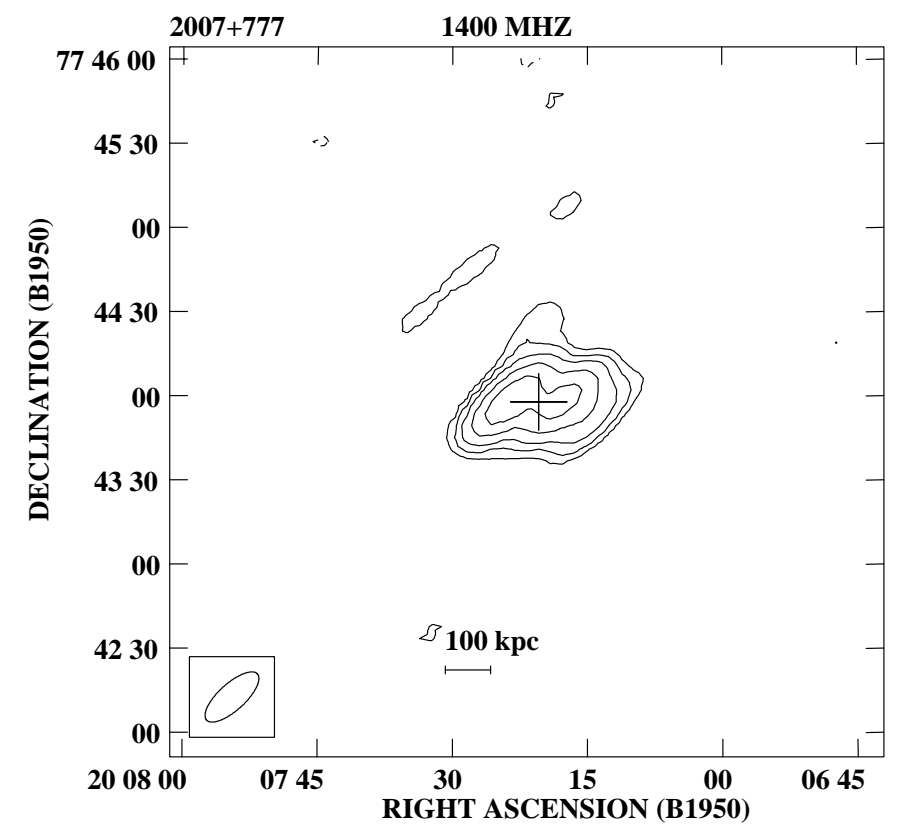

Fig. 30. 2007+777, WSRT, $1.40 \mathrm{GHz}$. The restoring beam is $24.0 \times 10.0$ arcsec in PA $-47^{\circ}$. The peak flux density is $17 \mathrm{mJy} /$ beam and the rms noise on the image is $0.25 \mathrm{mJy} /$ beam

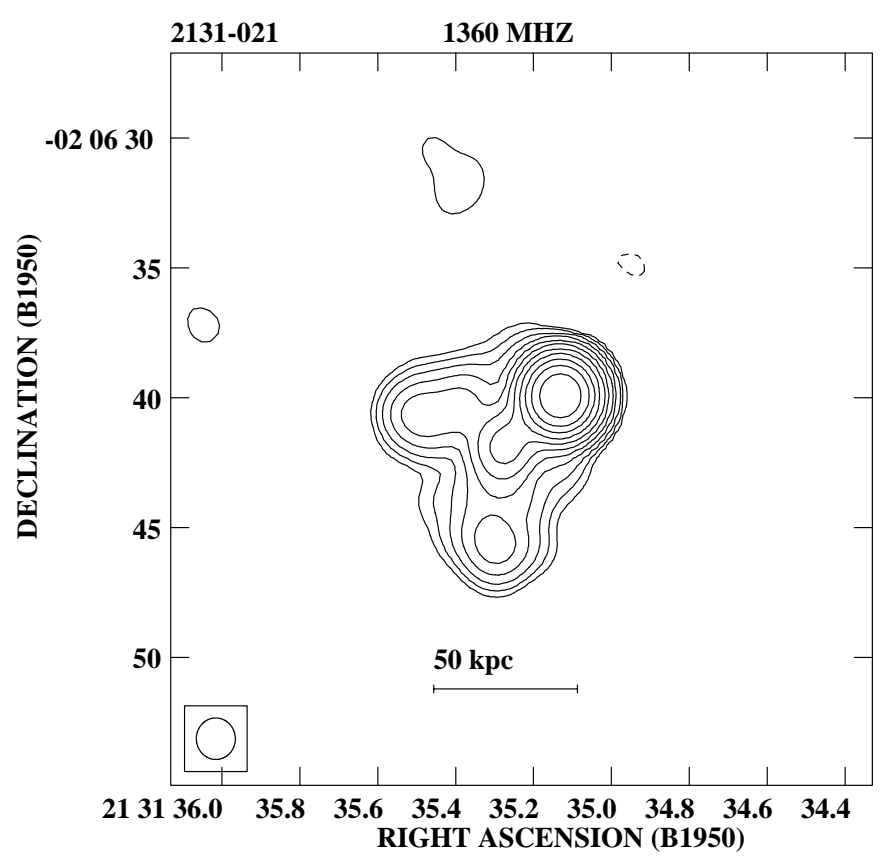

Fig. 31. 2131-021, VLA A configuration, $1.36 \mathrm{GHz}$. The restoring beam is $1.6 \times 1.5$ arcsec in PA $1^{\circ}$. The peak flux density is $1291 \mathrm{mJy} /$ beam and the rms noise on the image is $0.20 \mathrm{mJy} /$ beam

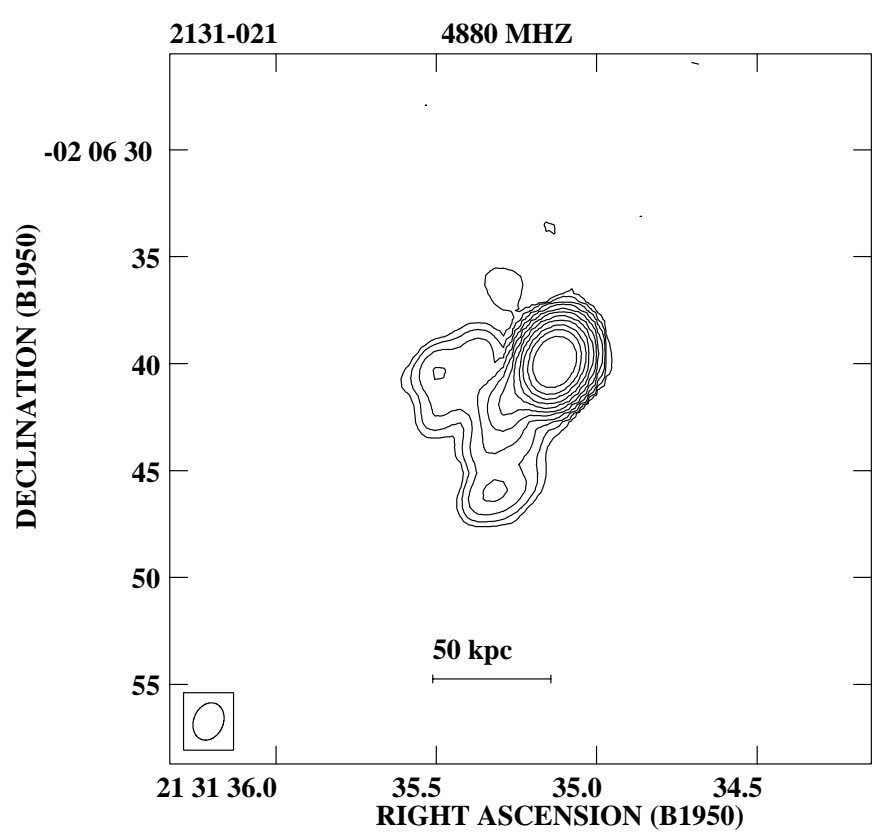

Fig. 32. 2131-021, VLA B configuration, $4.88 \mathrm{GHz}$. The restoring beam is $1.9 \times 1.4 \operatorname{arcsec}$ in $\mathrm{PA}-22^{\circ}$. The peak flux density is $982 \mathrm{mJy} /$ beam and the $\mathrm{rms}$ noise on the image is $0.20 \mathrm{mJy} /$ beam 


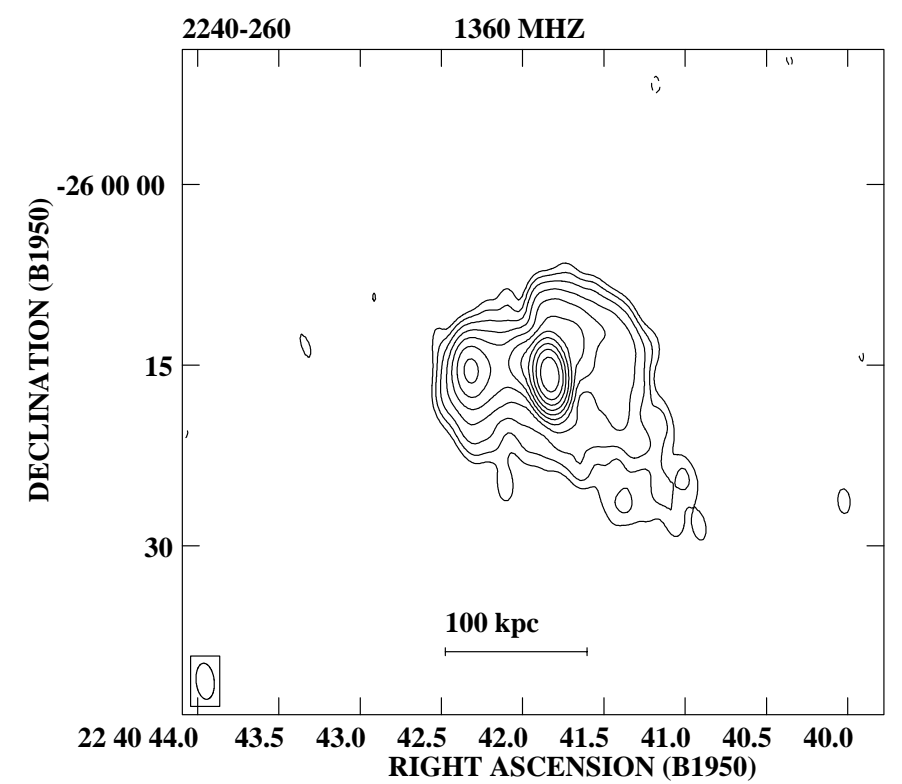

Fig. 33. 2240-260, VLA A configuration, 1.36 GHz. The restoring beam is $2.0 \times 1.0$ arcsec in PA $6^{\circ}$. The peak flux density is $813 \mathrm{mJy} /$ beam and the rms noise on the image is $0.08 \mathrm{mJy} /$ beam

leading to the NW and a rather bright component extending approximately $4^{\prime \prime}$ south of the core. The $4.88 \mathrm{GHz}$ image (Fig. 6) does not show clear secondary components, maybe just a clue of the southern one. The source is pointlike in the D array image. Previous observations (Perley 1982) do not reveal any extended structure in this source.

We remark that recent optical observations by Heidt et al. (1996) and Scarpa et al. (1999) show a number of companions within 3 arcsec from the BL Lac. The extended radio emission in Fig. 5 is aligned with the position of these companions. We cannot rule out that at least part of the extended radio emission we report in Table 3 is associated with any of these structures.

0426-380: this source has a short jet pointing to the NW visible only in the higher resolution images (Figs. 7 and 8). We find a flux density of the extended emission twice the value reported by Perley (1982).

0537-441: the low declination of the source resulted in a very elongated beam in N-S. Nevertheless in our highest resolution image (Fig. 9, A array, $1.36 \mathrm{GHz}$ ) 0537-441 shows a curved jet-like structure leading to the west. The radio source is slightly resolved also in the B array image at the same wavelength (not shown). The jet has been also detected at $4.88 \mathrm{GHz}$, but its surface brightness is lower (Fig. 10). Perley (1982) detects only the knot at the end of the jet.

0828+493: no extended emission has been detected for this source either in our WSRT image or in previous

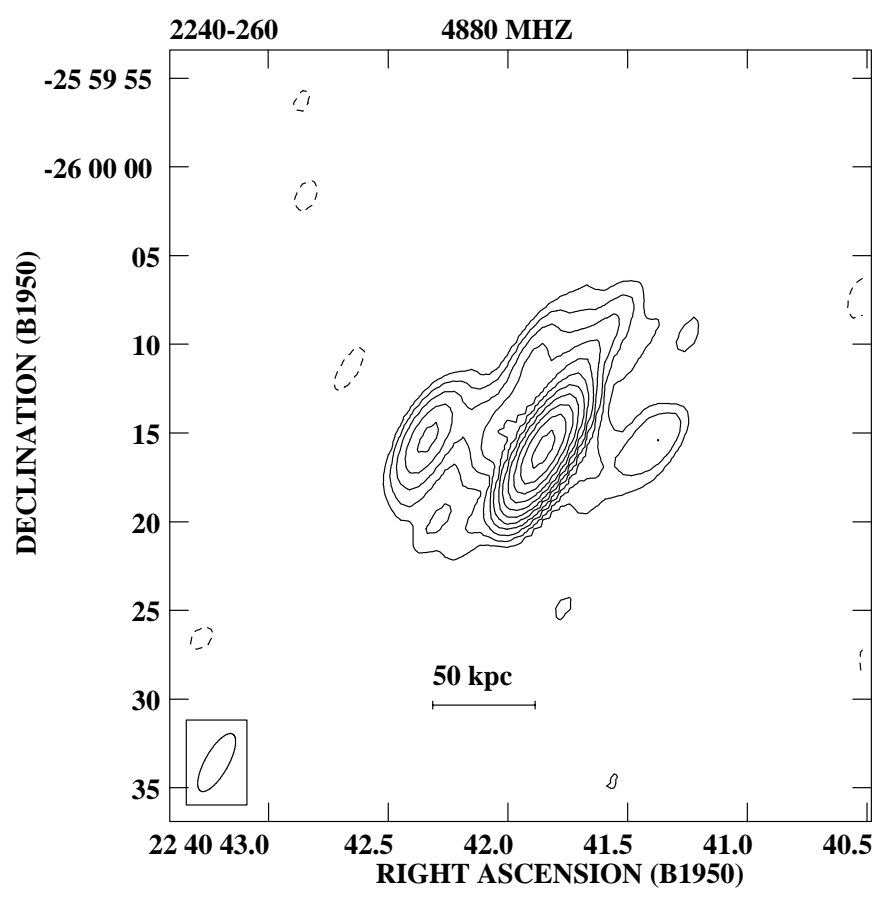

Fig. 34. 2240-260, VLA B configuration, $4.88 \mathrm{GHz}$. The restoring beam is $3.7 \times 1.3$ arcsec in PA $-29^{\circ}$. The peak flux density is $805 \mathrm{mJy} /$ beam and the rms noise on the image is $0.20 \mathrm{mJy} /$ beam

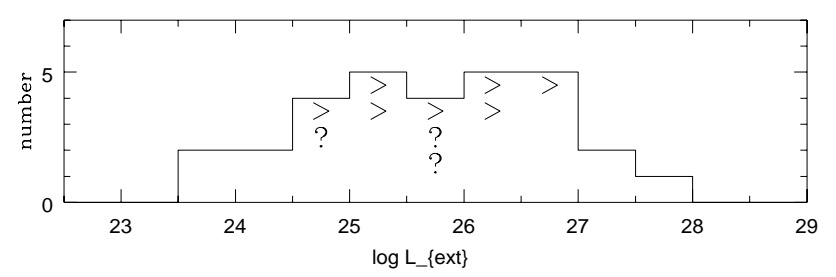

Fig. 35. Numeric distribution of the radio luminosity of the extended emission at $1.36 \mathrm{GHz}$ for the $1 \mathrm{Jy}$ sample, as from Table 3. The symbol " $>$ " is a lower limit to the luminosity, as derived from the minimum redshift. When the radio luminosity could not be determined with enough accuracy $(0823+033$, $0828+493$ and $1144-379$, see text), we used the symbol "?" to remark the uncertain estimate

VLA observations (Murphy et al. 1993). However, some extended emission has been recently revealed by the FIRST survey. We used this image (Fig. 11) to derive the numbers in Table 3.

0954+658: Kollgaard et al. (1992) detect a jet extending approximately $5^{\prime \prime}$ to the South. Our A array image at $1.36 \mathrm{GHz}$ (Fig. 12) and the $4.88 \mathrm{GHz} \mathrm{B}$ array image (Fig. 13) show an elongated structure directed to the SW and then bending to the south. No further extended emission has been detected in our B and D array data at $1.36 \mathrm{GHz}$.

1144-379: the radio image in the $L$ band is dominated by an unresolved component of $1.9 \mathrm{Jy}$. Perley 
Table 3. Observational data of the whole 1 Jy sample. The question marks in Cols. [2] through [7] indicate indicate a very uncertain value. Column [1] IAU name; [2] red shift: Stickel et al. (1993) for all sources but 0138-097 and $0454+844$ (Stocke \& Rector 1997), 0814+425 (Falomo et al. 1997) and 2131-021 (Drinkwater et al. 1997); [3] extended flux, where necessary scaled to $1.36 \mathrm{GHz}$; [4] Log of extended luminosity; [5] maximum diameter of the source in arcsec and [6] in kpc; [7] ratio core/extended flux density; [8] sidedness: "1" one-sided extended emission, "2" double-sided extended emission, "P" unresolved or barely resolved source; [9] references for the information given in this table: 1) this paper, 2) Murphy et al. (1993), 3) Antonucci et al. (1996), 4) Antonucci \& Ulvestad (1995), 5) Kollgaard et al. (1992), 6) Perlman \& Stocke (1994), 7) FIRST survey

\begin{tabular}{|c|c|c|c|c|c|c|c|c|}
\hline [1] & {$[2]$} & {$[3]$} & {$[4]$} & {$[5]$} & {$[6]$} & {$[7]$} & [8] & [9] \\
\hline Name & $z$ & $\begin{array}{c}S_{\text {ext }} \\
(\mathrm{mJy})\end{array}$ & $\begin{array}{r}\log L_{\text {ext }} \\
\left(\mathrm{W} \mathrm{Hz}^{-1}\right)\end{array}$ & $\begin{array}{c}\text { LAS } \\
(\operatorname{arcsec})\end{array}$ & $\begin{array}{l}\text { Size } \\
(\mathrm{kpc})\end{array}$ & $R$ & $S$ & Ref \\
\hline $0048-097$ & $>0.2$ & 176 & $>25.50$ & 24 & $>101$ & $<2.5$ & 1 & 1 \\
\hline $0118-272$ & $>0.557$ & 168 & $>26.40$ & 18 & $>133$ & $<3.0$ & 1 & 1 \\
\hline $0138-097$ & 0.733 & 50 & 26.13 & 23 & 186 & 6.8 & 2 & 1 \\
\hline $0235+164$ & 0.940 & 36 & 26.21 & 13 & 110 & 18.2 & 1 & 2 \\
\hline $0426-380$ & $>1.030$ & 86 & $>26.67$ & 6 & $>51$ & $<4.1$ & 1 & 1 \\
\hline $0454+844$ & $>1.34$ & $\ldots$ & $\ldots$ & $\ldots$ & $\ldots$ & $\ldots$ & $\ldots$ & $\ldots$ \\
\hline $0537-441$ & 0.896 & 220 & 26.95 & 13 & 109 & 8.2 & 1 & 1 \\
\hline $0716+714$ & $>0.2$ & 387 & $>25.85$ & 18 & $>76$ & $<0.87$ & 1 & 3 \\
\hline $0735+178$ & $>0.424$ & 24 & $>25.31$ & 10 & $>66$ & $<70.0$ & 1 & 2 \\
\hline $0814+425$ & $>0.6$ & 76 & $>26.12$ & 6 & $>46$ & 16.8 & 1 & 2 \\
\hline $0820+225$ & 0.951 & 700 & 27.51 & 12 & 101 & 1.3 & 1 & 2 \\
\hline $0823+033$ & $0.506 ?$ & 5 & $24.79 ?$ & 18 & $129 ?$ & 191.0 & 1 & 2 \\
\hline $0828+493$ & $0.548 ?$ & 25 & $25.56 ?$ & 42 & $331 ?$ & 10.0 & 2 & 7 \\
\hline $0851+202$ & 0.306 & 17 & 24.87 & 28 & 156 & 81.6 & 1 & 6 \\
\hline $0954+658$ & 0.367 & 34 & 25.33 & 6 & 37 & 14.7 & 1 & 1 \\
\hline $1144-379$ & 1.048 & $10 ?$ & $25.75 ?$ & $1 ?$ & $8 ?$ & $106.5 ?$ & $\mathrm{P}$ & 1 \\
\hline $1147+245$ & $>0.2$ & 50 & $>24.96$ & 34 & $>144$ & $<13.7$ & 2 & 1 \\
\hline $1308+326$ & 0.997 & 105 & 26.73 & 31 & 264 & 4.7 & 1 & 1 \\
\hline $1418+546$ & 0.152 & 47 & 24.69 & 75 & 259 & 13.5 & 1 & 1 \\
\hline $1514-241$ & 0.049 & 210 & 24.34 & 270 & 354 & 7.4 & 1 & 1 \\
\hline $1519-273$ & $>0.2$ & $\ldots$ & $\ldots$ & $\ldots$ & $\ldots$ & $\ldots$ & $\mathrm{P}$ & 1 \\
\hline $1538+149$ & 0.605 & 234 & 26.62 & 7 & 53 & 4.5 & 1 & 2 \\
\hline $1652+398$ & 0.033 & 95 & 23.65 & 75 & 68 & 14.2 & 2 & 1 \\
\hline $1749+096$ & 0.320 & $\ldots$ & $\ldots$ & $\ldots$ & $\ldots$ & $\ldots$ & $\ldots$ & $\ldots$ \\
\hline $1749+701$ & 0.770 & 12 & 25.55 & 3 & 24 & 32.2 & $?$ & 5 \\
\hline $1803+784$ & 0.684 & 43 & 26.00 & 56 & 444 & 27.0 & 1 & 1 \\
\hline $1807+698$ & 0.051 & 1010 & 25.06 & 222 & 302 & 1.2 & 2 & 1 \\
\hline $1823+568$ & 0.664 & 525 & 27.06 & 25 & 196 & 1.3 & $?$ & 2 \\
\hline $2005-489$ & 0.071 & $\ldots$ & $\ldots$ & $\ldots$ & $\ldots$ & $\ldots$ & $\ldots$ & $\ldots$ \\
\hline $2007+777$ & 0.342 & 41 & 25.35 & $28^{a}$ & $166^{a}$ & 21.1 & $2^{a}$ & 1 \\
\hline $2131-021$ & 1.285 & 182 & 27.20 & 9 & 77 & 3.7 & 1 & 1 \\
\hline $2200+420$ & 0.069 & 40 & 23.92 & 15 & 27 & 78.4 & 1 & 4 \\
\hline $2240-260$ & 0.774 & 333 & 26.99 & 26 & 212 & 1.6 & 2 & 1 \\
\hline $2254+074$ & 0.190 & 17 & 24.44 & 18 & 73 & 23.2 & 1 & 4 \\
\hline
\end{tabular}

Note: $a$. Value calculated from the image of Murphy et al. (1993).

(1982) reports an upper limit of $10 \mathrm{mJy}$ for any extended emission. The sources $1144-379$ is barely resolved even in the VLA A array images. A coarse estimate of the extended flux, through fitting the radio source with a point-like component and determining the residuals, gives a value of about 20 mJy. In Fig. 14 we show the VLA image in which the arcsecond core has not been restored (its position is marked by a cross). Given the low declination of the source, the $u v$-coverage is not adequate to allow a proper imaging of this additional component.
1147+245: we detect a diffuse component up to $15^{\prime \prime}$ to the South and another diffuse component located about $10^{\prime \prime}$ on the opposite side of the core (Fig. 15), basically in agreement with the image published by Antonucci \& Ulvestad (1985). Our measure of the extended emission flux density is about twice their value. In the $4.88 \mathrm{GHz}$ image (Fig. 16) only hints of the extended structures are visible.

1308+326: The VLA data of this source are from a different program, in which $1308+326$ was observed as 
secondary calibrator. This source shows a dominant component which is resolved in the SE direction, a secondary component $\sim 12^{\prime \prime}$ north, and a diffuse halo surrounding the entire structure (Fig. 17). Murphy et al. (1993) resolve the southern diffuse emission in a structure suggesting a helical jet. Our B array image reveals $\sim 30 \%$ more flux density in the extended structure than reported by Murphy et al. (1993).

1418+546: Murphy et al. (1993) found a component to the West of the compact core also present in the image from the FIRST survey (Fig. 18). Our WSRT observations (Fig. 19) show that this component is elongated to the south, with an extended flux density higher than reported by Murphy et al. or revealed by the FIRST image. The low resolution D array image (Fig. 20 ) is dominated by a point-like component; however extended and diffuse emission is detected all around, suggesting the presence of a halo with a total size of 4.5 arcmin similar to that observed in $1807+698$ (see below).

1514-241: Antonucci \& Ulvestad (1985) report a component $21^{\prime \prime}$ away from the core. Our A+B array image at $1.36 \mathrm{GHz}$ shows a jet emerging along the $\mathrm{SE}$ direction and bending towards $\mathrm{NE}$ after a dozen of arcseconds, for a total extent $\sim 55^{\prime \prime}$ (Fig. 21), also visible in the $4.88 \mathrm{GHz}$ image (Fig. 22). The image from the D array (Fig. 23) was obtained from the NVSS survey (Condon et al. 1998), and clearly shows a diffuse emission on the arcmin scale on the same side of the jet. The flux density of this component was added to the estimate from our $\mathrm{A}+\mathrm{B}$ array image.

1519-273: this is a very compact source, unresolved in our images at the resolution of $1^{\prime \prime}$. The source is still unresolved at the mas scale (O'Dea et al. 1991; Shen et al. 1997). Perley (1982) gives an upper limit of about $5 \mathrm{mJy}$ for any extended emission. We did not detect significant extended emission above $0.5 \mathrm{mJy} /$ beam, and we do not show any image for this source.

1652+398: Kollgaard et al. (1992) find a diffuse emission at $5 \mathrm{GHz}$ which is in agreement with the $75^{\prime \prime}$ wide halo we detect in our higher resolution B array image at 1.36 GHz (Fig. 24). Our 4.88 image shows instead only a small fraction of the extended emission visible in the $L$ band.

1803+784: Kollgaard et al. (1992) find a weak component $45^{\prime \prime}$ away from the core, while Antonucci et al. (1986) detect a diffuse emission around the core. In our image (Fig. 26) a jet-like structure is present connecting the secondary component to the core, and additional diffuse emission on the west side. The $4.88 \mathrm{GHz}$ image (not reported) shows only an unresolved core.
1807+698: Kollgaard et al. (1996) find the radio structure of this source, at arcsecond resolution, consisting of a $\sim 30^{\prime \prime}$ long jet extending from the core to the west direction. Wrobel \& Lind (1990) find a double lobed structure of total extension of $\sim 60^{\prime \prime}$, at $4.88 \mathrm{GHz}$ (VLA, $\mathrm{B}$ configuration). Our image from $\mathrm{A}+\mathrm{B}+\mathrm{D}$ configuration (Fig. 27) shows a diffuse halo of $\sim 220^{\prime \prime}$ of extension surrounding the core-jet structure in agreement with the morphology seen by Wrobel \& Lind (1990). The jet is clearly visible in our A array image at $1.36 \mathrm{GHz}$ (Fig. 28) and in the B array $4.88 \mathrm{GHz}$ image (Fig. 29).

2007+777: the WSRT image has not enough resolution to characterize the extended structure, which is better highlighted by the VLA observations of Murphy et al. (1993). However the total flux density of the extended emission in our WSRT image exceeds the measure from Murphy et al. by about 30\% (Fig. 30).

2131-021: the unresolved core is located at the NW edge of the radio emission (Fig. 31). Two jet like structures are oriented in $\mathrm{PA} \sim-90^{\circ}$ and $\mathrm{PA} \sim-170^{\circ}$; all this is reminiscent of NAT/WAT morphology. The B array image at $1.36 \mathrm{GHz}$ does not reveal any further extended emission and we can therefore consider that the total angular size of the extended radio emission is about $9^{\prime \prime}$. Observations of this source were made by Perley (1982) and he found an extended flux of about $50 \mathrm{mJy}$. Recently a VLA image in B configuration at $1.49 \mathrm{GHz}$ was published by Hutchings et al. (1998). The lower resolution of their observations did not allow to properly separate the core flux density from the tail-shaped extended emission yielding to underestimate the total extended flux density.

2240-260: in this object the unresolved core sits in the center of a diffuse extended emission which can be characterized by two misaligned and bent jet-like structures (Fig. 33). The total size of the extended emission is about $26^{\prime \prime}$ corresponding to $212 \mathrm{kpc}$ at the redshift of the host galaxy. No previous arcsecond scale observations have been found in the literature.

\section{Discussion and conclusions}

Unified Schemes Models consider the low-power FR I radio galaxies as the parent, unbeamed population of BL Lac objects. The boundary between FR I and the powerful FR II radio galaxies has been found to lie around $210^{25} \mathrm{~W} \mathrm{~Hz}^{-1}$ at $178 \mathrm{MHz}$ (Fanaroff \& Riley 1974). At higher frequencies, like at $1.36 \mathrm{GHz}$, the segregation in radio power is not as evident as at $178 \mathrm{MHz}$, and in the literature one commonly finds that $\sim 10^{24.5} \mathrm{~W} \mathrm{~Hz}^{-1}$ is considered the borderline. Indeed, it is well known (e.g. Bridle 1984,1987 ) that this boundary at $1.4 \mathrm{GHz}$ is 
not really sharp, although one can infer that most of the FR I radio galaxies are below that limit, while most of the FR II lie above. We therefore investigated the properties of the extended emission in the 1 Jy BL Lac sample, and in particular we studied the extended, unbeamed emission.

The monochromatic luminosities derived from our observations are often larger than those found in the literature; furthermore, for a few sources we have revealed extended structure previously unknown. Therefore this work is more complete and goes deeper than previous efforts in this direction.

In Fig. 35 we present a plot of the distribution of the luminosity of the extended, unbeamed emission for the 1 Jy BL Lac sample, as given in Table 3, once the arcsecond scale core has been subtracted out. We have used the measurements derived from our observations, completed with data taken from the literature (see Table 3). On this respect, we chose the better suited images/measurements in order to have about the same accuracy in the subtraction of the arcsecond core emission.

The unbeamed radio luminosities at $1.36 \mathrm{GHz}$ clearly extend beyond the limit (not really sharp at this frequency) of $10^{24.5} \mathrm{~W} \mathrm{~Hz}^{-1}$ separating FR I from FR II radio galaxies, with just a few objects with radio luminosities typical of the brightest FR II galaxies. In general the luminosity distribution of the whole sample seems rather smooth and covers a wide range of radio luminosities.

In a forthcoming paper we will discuss in detail our results, and we will compare the radio properties of the Radio Selected BL Lacs (i.e. the 1 Jy sample) with those of X-ray Selected BL Lacs (i.e. EMSS sample) and further with those of FR I and FR II radio galaxies.

\section{Summary}

We have presented interferometric radio data at 1.36, 1.66 and $4.8 \mathrm{GHz}$ with the VLA at A, B and D configurations, and at $1.4 \mathrm{GHz}$ with the WSRT, on 28 of 34 objects of the 1 Jy sample of BL Lac objects (Stickel et al. 1991). We have obtained high sensitivity radio images at the arcsec resolution, in order to evaluate the extended luminosity of these objects, most of which had poor or no radio data at this resolution. We found that most of the sources observed possess substantial extended emission, and often our flux densities exceed those previously reported into the literature. A few sources have unbeamed radio luminosities at $1.36 \mathrm{GHz}$ of the order of $10^{27} \mathrm{~W} \mathrm{~Hz}^{-1}$, supporting the hypothesis that the parent population of $\mathrm{BL}$ Lac objects is a mixture of FR I and FR II radio sources.

Acknowledgements. DD and MB acknowledge financial support from the European Union as an EU Fellow under contract CHBGCT920212. This work was partly supported by the Italian Ministry for University and Research (MURST) under grant Cofin98-02-32 The National Radio Astronomy Observatory is operated by Associated University Inc under contract with the National Science Foundation. The WSRT is operated by the Netherlands Foundation for Research in Astronomy (NFRA) with financial support by the Netherlands Organization for Scientific Research (NWO). We thank the referee, Travis Rector, whose comments significantly improved the paper.

\section{References}

Antonucci R.R.J., Ulvestad J.S., 1985, ApJ 294, 158

Antonucci R.R.J., Hickson P., Olszewsky E.W., Miller J.S., 1986, AJ 92, 1

Baars J.W., Genzel R., Pauliny-Toth I.I.K., Witzel A., 1977, A\&A 61,99

Becker R.H., White R.L., Helfand D.J., 1995, ApJ 450, 559

Blandford R.D., Rees M.J., 1978, in Pittsburgh Conference on BL Lac Objects, Wolfe A.M. (ed.). University of Pittsburgh, Pittsburgh, p. 328

Bridle A.H., 1984, AJ 89, 979

Bridle A.H., 1987, in Active Galactic Nuclei, Lecture Notes in Physics, No. 307, Miller H.R., Wiita P.J. (eds.). Springer, New York, p. 329

Condon J.J., Cotton W.D., Greisen E.W., et al., 1998, AJ 115, 1693

Drinkwater M.J., Webster R.L., Francis P.J., et al., 1997, MNRAS 284, 85

Falomo R., Urry C.M., Pesce J.E., et al., 1997, ApJ 476, 113

Fanaroff B.L., Riley J.M., 1974, MNRAS 167, 31

Heidt J., Nilsson K., Pursimo T., et al., 1996, A\&A 312, L13

Hutchings J.B., Dewey A., Chaytor D., et al., 1998, PASP 110, 111

Kollgaard R.I., Wardle J.F.C., Roberts D.H., Gabuzda D.C., 1992, AJ 104, 1687

Kollgaard R.J., Palma C., Laurent-Muehleisen S.A., Feigelson E.D., 1996, ApJ 465, 115

Murphy D.W., Browne W.A., Perley R.A., 1993, MNRAS 264, 298

O'Dea C.P., Baum S.A., Stanghellini C., 1991, ApJ 380, 66

O'Dea C.P., Baum S.A., Stanghellini C., et al., 1992, AJ 104, 1320

Patnaik A.R., Browne I.W.A., King L.J., et al., 1993, MNRAS 261,435

Patnaik A.R., Porcas R.W., Browne I.W.A., 1995, MNRAS $274, \mathrm{~L} 5$

Perley R.A., 1982, AJ 87, 859

Perlman E.S., Stocke J.T., 1994, AJ 108, 56

Scarpa R., Urry C.M., Falomo R., et al., 1999, ApJ 521, 134

Shen Z.-Q., Wan T.-S., Moran J.M., et al., 1997, AJ 114, 1999

Stanghellini C., Dallacasa D., Bondi M., Della Ceca R., 1997, A\&A 325, 911

Stickel M., Fried J.W., Kühr H., et al., 1991, ApJ 374, 431

Stickel M., Fried J.W., Kühr H., 1993, A\&AS 98, 393

Stocke J.T., Rector T.A., 1997, ApJ 489L, 17

Urry C.M., Padovani P., 1995, PASP 107, 803

Wardle J.F.C., Moore R.L., Angel J.R.P., 1984, ApJ 279, 93

Wrobel J.M., Lind K.M., 1990, ApJ 348, 135 